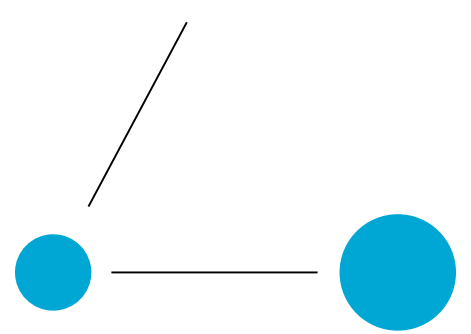

A N N A L E S HENRI LEBESGUE

\title{
THE TETRAHEDRON AND AUTOMORPHISMS OF ENRIQUES AND COBLE SURFACES OF HESSIAN TYPE
}

LE TÉTRAÈdRE, ET LES AUTOMORPHISMES DES SURFACES DE ENRIQUES ET DE COBLE DE TYPE HESS IENNES

To the memory of Ernest Borisovich Vinberg

Abstract. - Consider a cubic surface satisfying the mild condition that it may be described in Sylvester's pentahedral form. There is a well-known Enriques or Coble surface $S$ with K3 cover birationally isomorphic to the Hessian surface of this cubic surface. We describe the nef cone and $(-2)$-curves of $S$. In the case of pentahedral parameters $(1,1,1,1, t \neq 0)$ we compute the automorphism group of $S$. For $t \neq 1$ it is the semidirect product of the free product $(\mathbb{Z} / 2)^{* 4}$ and the symmetric group $\mathfrak{S}_{4}$. In the special case $t=\frac{1}{16}$ we study the action of $\operatorname{Aut}(S)$ on an invariant smooth rational curve $C$ on the Coble surface $S$. We describe the action and its image, both geometrically and arithmetically. In particular, we prove that

Keywords: Enriques surfaces, Coble surfaces, Automorphism groups, Hyperbolic geometry. 2020 Mathematics Subject Classification: 14J50, 20F65, 20F67.

DOI: https://doi.org/10.5802/ahl.57

(*) First author supported by Simons Foundation Collaboration Grant 429818. Second author supported by JSPS Grant-in-Aid (S), No. 25220701. 
$\operatorname{Aut}(S) \rightarrow \operatorname{Aut}(C)$ is injective in characteristic 0 and we identify its image with the subgroup of $\mathrm{PGL}_{2}$ coming from the isometries of a regular tetrahedron and the reflections across its facets.

RÉsumé. - Donnons-nous une surface cubique et faisons l'hypothèse, faible, que cette surface peut être décrite sous forme pentaèdrique de Sylvester. Il est bien connu que l'on peut trouver une surface de Enriques ou de Coble $S$ dont un revêtement double est une surface K3 birationnellement isomorphe à la hessienne de cette surface cubique. Nous décrivons le cône nef et les -2-courbes de $S$. Si les paramètres pentaèdriques sont $(1,1,1,1, t \neq 0)$ nous calculons le groupe d'automorphismes de $S$. Lorsque $t \neq 1$, c'est le produit semi-direct du produit libre $(\mathbb{Z} / 2)^{* 4}$ et du groupe symétrique $\mathfrak{S}_{4}$. Dans le cas particulier $t=\frac{1}{16}$ nous étudions l'action de $\operatorname{Aut}(S)$ sur une courbe rationnelle, lisse et invariante $C$ de la surface de Coble $S$. Nous décrivons l'action et son image, de manière géométrique et arithmétique à la fois. En particulier, nous montrons que l'homomorphisme $\operatorname{Aut}(S) \rightarrow \operatorname{Aut}(C)$ est injectif en caractéristique 0 et nous identifions son image au sous-groupe de $\mathrm{PGL}_{2}$ associé aux isométries d'un tétraèdre régulier et aux réflexions le long de ses faces.

\section{Introduction}

Let $\mathrm{UC}(4)$ be the universal Coxeter group on 4 generators, i.e. a free product of four groups of order 2. The permutation group $\mathfrak{S}_{4}$ acts naturally on it. Let $G=\mathrm{UC}(4) \rtimes \mathfrak{S}_{4}$ be the semi-direct product. In this article we realize the group $G$ in several ways:

(1) $G$ is the group of automorphisms of every Enriques and Coble surface in a certain 1-parameter family.

(2) $G$ acts on an invariant smooth rational curve on a particular rational surface in this family, faithfully when in characteristic 0 .

(3) $G$ is a discrete group of motions of hyperbolic space $H^{9}$.

(4) $G$ is the (nondiscrete) group of isometries of 3-dimensional Euclidean space generated by the isometries of a regular tetrahedron and the reflections across its facets.

(5) $G$ is the group of $\mathbb{Z}\left[\frac{1}{3}\right]$-valued points of an algebraic group scheme over $\mathbb{Z}$ coming from automorphisms of Hamilton's quaternion algebra.

(6) $G$ is maximal among discrete subgroups with finite covolume in $\mathrm{PGL}_{2}\left(\mathbb{Q}_{3}\right)$, where $\mathbb{Q}_{3}$ is the field of 3-adic rationals.

The geometrical motivation for the study of this remarkable group is a question discussed by Arthur Coble in his book [Cob82, p. 226-227] and a later paper [Cob39]: given a group of birational automorphisms of an algebraic surface $S$ over an algebraically closed field $\mathbb{k}$ that leaves a rational curve $C$ on it invariant, what are the image and kernel of the restriction map to $\operatorname{Bir}(C) \cong \mathrm{PGL}_{2}$ ? Interest in this problem was recently resurrected in works of Dinh and Oguiso [DO19] and Lesieutre [Lei18]. In our situation, $\mathbb{k}$ has characteristic $\neq 2,3$ and $S$ is the surface obtained by blowing up all ten double points of a certain plane rational curve of degree 6 that admits $\mathfrak{S}_{4}$ as its group of projective symmetries (see [Dol18, Section 5.4]). This surface is a special case of a rational Coble surface, whose definition we will recall in the next section. The curve $C \subset S$ is the proper transform of this sextic. 
Another description of $S$ begins with the Hessian surface $H$, of a cubic surface having six Eckardt points (points where three lines meet) and one ordinary node. There is a birational involution of $\mathbb{P}^{3}$ that acts freely away from the node, and $S$ is the minimal resolution of the quotient of $H$ by it. We will show that the group of automorphisms of $S$ is isomorphic to $G$, via a homomorphism that identifies natural generators of $G=\mathrm{UC}(4) \rtimes \mathfrak{S}_{4}$ with natural generators of $\operatorname{Aut}(S)$. This allows us to deduce that the restriction homomorphism $G \rightarrow \mathrm{PGL}_{2}(\mathbb{k})$ is faithful when working in characteristic 0 . This faithfulness is appealing and suggestive, but does not say anything definite about Coble's original problem, because UC(4) $\rtimes \mathfrak{S}_{4}$ is much smaller than the automorphism group of a general Coble surface, which is a lattice in $\mathrm{SO}(9,1)$ by [CD89, Theorem 2.10.1].

The cubic surface that gives rise to this Coble surface $S$ is the $t=\frac{1}{16}$ member of the following one-parameter family of cubic surfaces

$$
y_{0}+\cdots+y_{4}=y_{0}^{3}+\cdots+y_{3}^{3}+t y_{4}^{3}=0
$$

where $t \neq 0$. This family is also projectively isomorphic to the pencil of cubic surfaces with $\mathfrak{S}_{4}$-symmetry of type $\mathrm{V}$ from Table 9.5.9 from [Dol12] ${ }^{(1)}$. The minimal resolution $S_{t}$ of the quotient of the Hessian surface is a Coble surface if $t \in\left\{\frac{1}{4}, \frac{1}{16}\right\}$, or if $t=1$ and char $\mathbb{k}=5$. Otherwise it is an Enriques surface. We compute the group of automorphisms of $S_{t}$ and obtain the amazing fact that it does not depend on the parameter $t \neq 1$ and is isomorphic to the group $G .^{(2)}$ The exceptional case $t=1$ corresponds to the Clebsch diagonal cubic surface. In this case, both $S_{t}$ and the cubic surface have automorphism group $\mathfrak{S}_{5}$, and $S_{t}$ has type VI in Kondo's classification of complex Enriques surfaces with finite automorphism group [Kon86].

Our strategy for working out $\operatorname{Aut}\left(S_{t}\right)$ is to use known automorphisms coming from the projections of the Hessian surface from its 10 nodes to build a concrete model for the real nef cone $\operatorname{Nef}_{\mathbb{R}}\left(S_{t}\right)$. Then we use the shape of this cone to show that these known automorphisms generate $\operatorname{Aut}\left(S_{t}\right)$. The nef cone is (the cone in $\mathbb{R}^{10}$ over) a polytope in hyperbolic 9 -space, which usually has infinitely many facets. (In the Coble case, $\operatorname{Nef}_{\mathbb{R}}\left(S_{t}\right)$ has dimension larger than 10 . But we identify a 10-dimensional slice of it on which $\operatorname{Aut}\left(S_{t}\right)$ acts faithfully. By restricting attention to that slice, the Coble and Enriques cases become uniform.) We are nevertheless able to give a useful description of it in Theorem 3.2, for surfaces arising from the Hessian surfaces of an arbitrary Sylvester non-degenerate cubic surface. It seems reasonable to hope that this description will enable the computation of their automorphism groups in the same generality. For a Sylvester nondegenerate nonsingular cubic surface with no Eckardt points this has been done by other methods by I. Shimada [Shi19].

\section{Acknowledgements}

The authors thank Shigeru Mukai for stimulating discussions about the automorphism groups of Enriques surfaces arising from Hessian surfaces during their

${ }^{(1)}$ There is a misprint in the formula: the term $a t_{1} t_{2} t_{3}$ must be added.

${ }^{(2)}$ Although the Picard lattice of the K3-cover of an Enriques surface from the family does not depend on the parameter, it is still an amazing fact. 
stay at RIMS. We are grateful to the referees for their careful reading and helpful suggestions.

\section{Enriques and Coble surfaces of Hessian type}

We work over an algebraically closed field $\mathbb{k}$ of characteristic $\neq 2,3$. Although some of the references in the paper refer to sources which work over the field of complex numbers, all the proofs of what we need extend to our case. This section develops the situation of [Dol18] in a manner which treats the Enriques and Coble cases uniformly.

Let $V_{3}$ be a cubic surface in $\mathbb{P}^{3}$ given by equation $F\left(x_{0}, x_{1}, x_{2}, x_{3}\right)=0$ and $H \subset \mathbb{P}^{3}$ be its Hessian surface, given by the determinant of the matrix of second partial derivatives of $F$. We call $V_{3}$ Sylvester non-degenerate if $F$ can be written as the sum of the cubes of five linear forms, any four of the linear forms being linearly independent. A well-known theorem of Sylvester [Dol12, Theorem 9.4.1] asserts that a general cubic surface is Sylvester non-degenerate and that a Sylvester nondegenerate cubic surface uniquely determines the linear forms, up to cube roots of unity and a common scaling factor. It is more convenient to work with certain multiples of the linear forms. Namely, up to a common factor, they have unique nonzero multiples $L_{0}, \ldots, L_{4}$ with the property $\sum_{a=0}^{4} L_{a}=0$. We define $\lambda_{0}, \ldots, \lambda_{4}$ by the relation $F=\sum_{a=0}^{4} \lambda_{a} L_{a}^{3}$. The $\lambda_{a}$ are nonzero by Sylvester nondegeneracy.

Using the map $\mathbb{P}^{3} \rightarrow \mathbb{P}^{4}$ defined by

$$
\left(x_{0}: x_{1}: x_{2}: x_{3}\right) \mapsto\left(L_{0}\left(x_{0}, x_{1}, x_{2}, x_{3}\right): \cdots: L_{4}\left(x_{0}, x_{1}, x_{2}, x_{3}\right)\right)
$$

we embed $V_{3}$ and $H$ into $\mathbb{P}^{4}$. Their images are defined by

$$
\sum_{a=0}^{4} y_{a}=\sum_{a=0}^{4} \lambda_{a} y_{a}^{3}=0 \quad \text { and } \quad \sum_{a=0}^{4} y_{a}=\sum_{a=0}^{4} \frac{1}{\lambda_{a} y_{a}}=0
$$

respectively. The last sum is shorthand for the quartic polynomial got by clearing denominators. When speaking of $\mathbb{P}^{3}$ we will always mean the hyperplane $\sum_{a=0}^{4} y_{a}=0$. The intersections of this hyperplane with the hyperplanes $y_{a}=0$ are called the faces of the Sylvester pentahedron, and their pairwise and triple intersections are called the edges and vertices of the pentahedron. Each face contains four edges, which form the intersection of that face with the Hessian. We write $L_{a b}$ for the edge $y_{a}=y_{b}=0$ and $P_{a b}$ for the vertex defined by $y_{c}=0$ for all $c \neq a, b . P_{a b}$ lies in $L_{c d}$ if and only if $\{a, b\} \cap\{c, d\}=\emptyset$. The lines and vertices form an abstract symmetric Desargues configuration $\left(10_{3}\right)$; see Figure 2.1.

The singularities of the Hessian surface are mild:

Lemma 2.1. - Let $V_{3}$ be a Sylvester non-degenerate cubic surface. The vertices of the pentahedron are ordinary double points (nodes) of the Hessian surfaces $H$. The singular points of $V_{3}$ coincide with the remaining singular points of $H$, none of which lie in the faces of the pentahedron. Each such point is an ordinary double point of $V_{3}$ and an ordinary double point of $H$. 


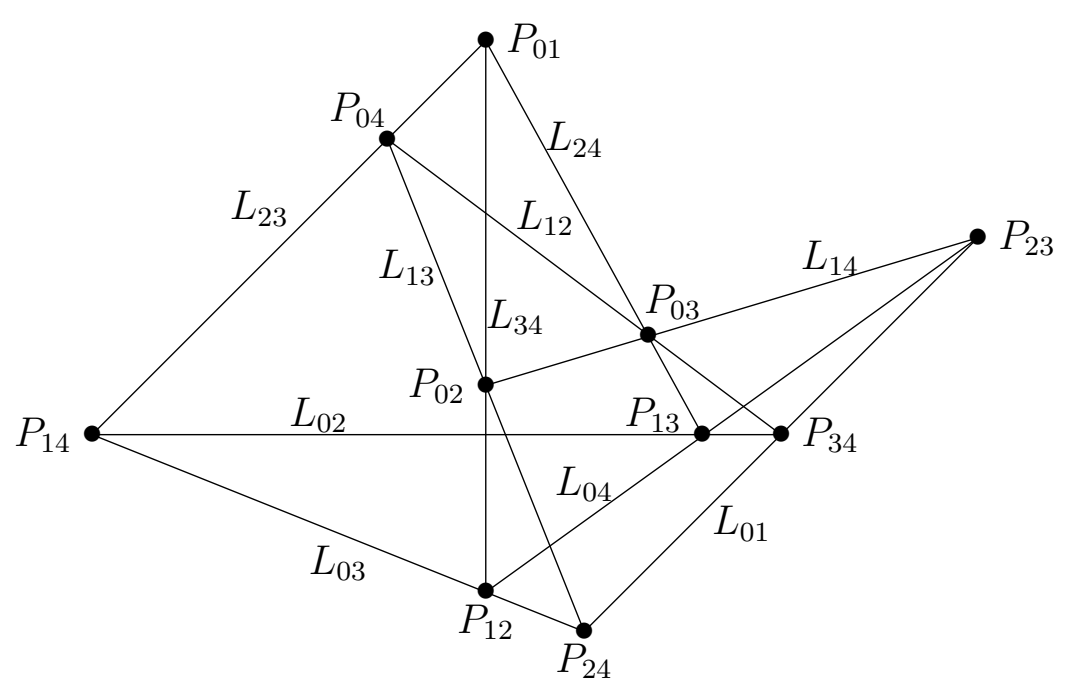

Figure 2.1. Sylvester pentahedron

Proof. - We clear the denominators of $\sum_{a} 1 / \lambda_{a} y_{a}$ to write it properly as a quartic hypersurface, and evaluate its $2^{\text {nd }}$ derivative matrix at the point $(1,-1,0,0,0)$ of affine space lying over a vertex. All entries vanish except the lower right $3 \times 3$ submatrix

$$
-\lambda_{0} \lambda_{1}\left(\begin{array}{ccc}
0 & \lambda_{2} \lambda_{3} & \lambda_{2} \lambda_{4} \\
\lambda_{2} \lambda_{3} & 0 & \lambda_{3} \lambda_{4} \\
\lambda_{2} \lambda_{4} & \lambda_{3} \lambda_{4} & 0
\end{array}\right)
$$

which has nonzero determinant. The nullspace of this quadratic form is spanned by the radial direction $(1,-1,0,0,0)$ in affine space and a vector $(1,0,0,0,0)$ transverse to the hyperplane $\sum_{a} y_{a}=0$. Therefore the local defining equation for $H$ in $\mathbb{P}^{3}$ has nondegenerate second derivative matrix at the vertex. This is the definition of a node.

Now let us find the singular points of $H$ away from the vertices of the pentahedron. Plugging in $y_{i}=0$ in the equation of $H$, we find that its section by the face of the pentahedron is a complete quadrilateral of the edges lying in the face. Its singular points are the six vertices. So no more singular points satisfy $y_{i}=0$, and we may take $\sum_{a=0}^{4} y_{a}=\sum_{a=0}^{4} 1 / \lambda_{a} y_{a}=0$ as the defining equations. The singular points are where the jacobian matrix

$$
\left(\begin{array}{ccccc}
\lambda_{0} & \lambda_{1} & \lambda_{2} & \lambda_{3} & \lambda_{4} \\
1 / \lambda_{0} y_{0}^{2} & 1 / \lambda_{1} y_{1}^{2} & 1 / \lambda_{2} y_{2}^{2} & 1 / \lambda_{3} y_{3}^{2} & 1 / \lambda_{4} y_{4}^{2}
\end{array}\right)
$$

has rank equal to 1 . This shows that the singular points of $H$ are given by the additional conditions $\lambda_{a} y_{a}^{2}=\lambda_{b} y_{b}^{2}$ for all $a, b$. Regarding $\left(y_{0}, \ldots, y_{4}\right)$ as a point of $\mathbb{A}^{5}$ lying over one of these singularities, the second derivative matrix of the polynomial $\sum 1 / \lambda_{i} y_{i}$ is a scalar multiple of the diagonal matrix $\operatorname{diag}\left[1 / y_{0}, \ldots, 1 / y_{4}\right]$. Again we regard this as a bilinear form on the tangent space of $\mathbb{A}^{5}$ at this point. To show that the singularity is an ordinary node, it is enough to show that this form's restriction to the coordinate-sum zero subspace has null space no larger than the radial direction 
in $\mathbb{A}^{5}$. This is immediate: if $\left(c_{0}, \ldots, c_{4}\right)$ lies in the null space, then orthogonality to the vectors like $(1,-1,0,0,0)$ forces $c_{a} / y_{a}=c_{b} / y_{b}$ for all $a, b$.

The corresponding analysis for $V_{3}$ turns out to be exactly the same calculation. In particular, its singular points are defined by the same conditions $\lambda_{a} y_{b}^{2}=\lambda_{b} y_{a}^{2}$. The only difference is that $V_{3}$ has no singularities in the faces of the pentahedron.

Since singularities of $H$ away from the vertices usually don't exist, and are nodes when they do, we call them the new nodes. We will write $k$ for the number of new nodes.

Because the Hessian $H$ is a quartic surface whose singularities are ordinary nodes, its minimal resolution $X$ is a K3 surface. We write $E_{a b}$ for the exceptional curve over $P_{a b}$. Because $P_{a b}$ is an ordinary node, $E_{a b}$ is a $(-2)$-curve, meaning a smooth rational curve with self-intersection -2 . We denote the proper transforms of the $L_{a b}$ by the same notation $L_{a b}$. The ten curves $E_{a b}$ (resp. $L_{a b}$ ) are disjoint. By the definition of the pentahedron, $L_{a b}$ contains $P_{c d}$ if and only if $\{a, b\}$ and $\{c, d\}$ are disjoint. Therefore

$$
L_{a b} \cdot E_{c d}= \begin{cases}1 & \text { if }\{a, b\} \cap\{c, d\}=\emptyset \\ 0 & \text { otherwise }\end{cases}
$$

The birational involution of $\mathbb{P}^{4}$ defined by the formula

$$
\sigma:\left(y_{0}: \cdots: y_{4}\right) \mapsto\left(\frac{1}{\lambda_{0} y_{0}}: \cdots: \frac{1}{\lambda_{4} y_{4}}\right)
$$

restricts to a birational self-map of $H$. This restriction is biregular on the complement of the faces of the pentahedron. The fixed points of $\sigma$ in this open set coincide with the new nodes, since the condition $\left[y_{0}: \cdots: y_{4}\right]=\left[1 / \lambda_{0} y_{0}: \cdots: 1 / \lambda_{4} y_{4}\right]$ is the same as the condition $\lambda_{a} y_{a}^{2}=\lambda_{b} y_{b}^{2}$ describing the new nodes. We also write $\sigma$ for the corresponding self-map of $X$, which is biregular. (Every birational map from one K3 surface to another is biregular). One can check that $\sigma$ swaps each $E_{a b}$ with the disjoint curve $L_{a b}$. It follows that $\sigma$ acts freely on $X$, away from the exceptional divisors over the new nodes.

The exceptional divisor over a new node is a $(-2)$-curve, got by blowing up once. Also, each new node is an isolated fixed point under the action of $\sigma$ on $\mathbb{P}^{4}$, so $\sigma$ acts by negation on the tangent space there. It follows that $\sigma$ acts trivially on the fibers over the new nodes. Therefore the quotient surface $S=X /\langle\sigma\rangle$ is smooth. $S$ is the main object of interest in this paper. We write $f: X \rightarrow S$ for the quotient map, $\mathcal{E}$ for the sum of the exceptional divisors over the new nodes, and $\mathcal{C}$ for the branch divisor in $S$ (the image of $\mathcal{E}$ ). This branch divisor consists of $k$ disjoint smooth rational curves with self-intersection -4 . Also, we write $U_{a b}$ for the common image in $S$ of $E_{a b}$ and $L_{a b}$. Since $f: X \rightarrow S$ is a degree 2 cover, the usual properties of intersection theory give

$$
\begin{aligned}
U_{a b} \cdot U_{c d} & =\frac{1}{2}\left(L_{a b}+E_{a b}\right) \cdot\left(L_{c d}+E_{c d}\right)=\frac{1}{2}\left(L_{a b} \cdot E_{c d}+L_{c d} \cdot E_{a b}\right) \\
& = \begin{cases}1 & \text { if }\{a, b\} \cap\{c, d\}=\emptyset \\
0 & \text { otherwise }\end{cases}
\end{aligned}
$$


where we used (2.1) in the last step. That is, the $U_{a b}$ are $(-2)$-curves which intersect according to the Petersen graph, whose symmetry group is the symmetric group $\mathfrak{S}_{5}$. See Figure 2.2.

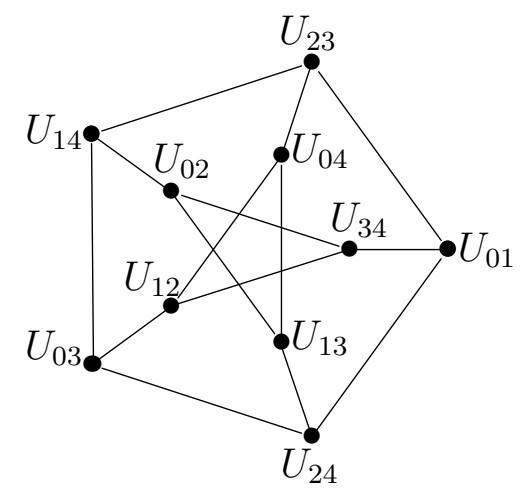

Figure 2.2. The Petersen graph

We recall that a Coble surface means a smooth rational surface whose anticanonical system is empty but whose bi-anticanonical system is not (see [DZ01]). They are important because they occur as degenerations of Enriques surfaces.

Lemma 2.2. - If there are no new nodes then $S$ is an Enriques surface. Otherwise, $S$ is a Coble surface, and $\mathcal{C}$ is the unique effective bi-anticanonical divisor on $S$.

Proof. - It is standard that the quotient of a K3 surface by a fixed-point-free involution is an Enriques surface. So suppose $\sigma$ has fixed points. The Hurwitz-type formula

$$
0=2 K_{X}=2\left(f^{*}\left(K_{S}\right)+\mathcal{E}\right)=f^{*}\left(2 K_{S}+\mathcal{C}\right)
$$

shows that $2 K_{S}+\mathcal{C}$ is numerically trivial, which is to say that $\mathcal{C}$ is numerically bi-anticanonical. If there were an effective bicanonical divisor, then its sum with $\mathcal{C}$ would be numerically trivial, which is absurd. Therefore $\left|2 K_{S}\right|=\emptyset$. Together with $H^{1}\left(S, \mathcal{O}_{S}\right)=0$, which follows from $H^{1}\left(X, \mathcal{O}_{X}\right)=0$, this lets us apply Castelnuovo's rationality criterion to deduce that $S$ is rational. By this rationality, numerical equivalence and linear equivalence of linear systems are equivalent. So $\mathcal{C}$ is a bianticanonical divisor. There are no other effective bi-anticanonical divisors because the components of $\mathcal{C}$ are disjoint with negative self-intersection. The only candidate for an effective divisor in $\left|-K_{S}\right|$ is $\frac{1}{2} \mathcal{C}$, but this is not a divisor, so $\left|-K_{S}\right|=\emptyset$. This proves that $S$ is a Coble surface.

(Another approach to the rationality of $S$ is to use the fact that the quotient of a K3 surface by an involution is birationally an Enriques surface, rational surface or a K3 surface. The last case happens if and only if the involution has eight isolated fixed points. So, in our case $S$ must be a rational surface.)

One of the goals of this paper is to understand $\operatorname{Aut}(S)$. We will use some elliptic fibrations $\left|G_{a b}\right|$ of $S$ in order to show that $\operatorname{Aut}(S)$ acts faithfully on a certain lattice $\Lambda$ in $\operatorname{Num}_{\mathbb{Q}}(S)$. To construct these elliptic fibrations we begin with some elliptic fibrations of $X$. 
Consider the pencil of planes in $\mathbb{P}^{3}$ that contain $L_{a b}$. Each plane meets $H$ in $L_{a b}$ and a plane cubic curve. The total transforms in $X$ of these residual cubics form a pencil of elliptic curves, which we identify with the elliptic fibration $X \rightarrow \mathbb{P}^{1}$ it defines. We write $\left|\widetilde{G}_{a b}\right|$ for this elliptic fibration and also the corresponding linear system. (This is harmless by the completeness of the pencil as a linear system. It is complete because every member of the linear system has trivial intersection number with every member of the pencil.) By taking the plane to be the face $y_{a}=0$ of the Sylvester pentahedron, one can express $\left|\widetilde{G}_{a b}\right|$ as

$$
\left|\widetilde{G}_{a b}\right|=\left|L_{a c}+L_{a d}+L_{a e}+E_{b c}+E_{b d}+E_{b e}\right|
$$

where $\{c, d, e\}=\{0, \ldots, 4\}-\{a, b\}$. This particular fiber has type $I_{6}$ in Kodaira's notation.

Lemma 2.3. - Each fibration $\left|\widetilde{G}_{a b}\right|$ is $\sigma$-invariant in the sense that $\sigma$ permutes its fibers. It permutes them nontrivially.

Proof. - The divisors $E_{c d}$ and $L_{c d}$ are swapped by $\sigma$, for each pair $c, d$. Therefore $\sigma$ sends

$$
\begin{array}{ll} 
& L_{a c}+L_{a d}+L_{a e}+E_{b c}+E_{b d}+E_{b e} \\
\text { to } \quad & E_{a c}+E_{a d}+E_{a e}+L_{b c}+L_{b d}+L_{b e},
\end{array}
$$

which is a different fiber of $\left|\widetilde{G}_{a b}\right|$, coming from the hyperplane section $y_{b}=0$. So $\sigma$ preserves the linear system and acts nontrivially on it.

The fibers (2.3) have common image

$$
F_{a b}:=U_{a c}+U_{a d}+U_{a e}+U_{b c}+U_{b d}+U_{b e}
$$

under $X \rightarrow S$. We write $\left|G_{a b}\right|$ for its linear system on $S$. This is pencil of elliptic curves for the same reason that $\left|\widetilde{G}_{a b}\right|$ is, so we also write $\left|G_{a b}\right|$ for the corresponding elliptic fibration. The projection $X \rightarrow S$ sends the class of a fiber of $\left|\widetilde{G}_{a b}\right|$ to the class of a fiber of $\left|G_{a b}\right|$.

Now we define the lattice $\Lambda$ on which $\operatorname{Aut}(S)$ will act faithfully. It was first introduced by S. Mukai in his unpublished work on automorphisms of Coble surfaces, so we call it the Coble-Mukai lattice. ${ }^{(3)}$ Let $C_{1}, \ldots, C_{k}$ be the components of the bi-anticanonical divisor $\mathcal{C}$. Let $\operatorname{Num}(S)^{\prime}$ be the lattice in $\operatorname{Num}_{\mathbb{Q}}(S)$ generated by $\operatorname{Num}(S)$ and $\frac{1}{2} C_{1}, \ldots, \frac{1}{2} C_{k}$. Let $\Lambda$ be the orthogonal complement of $C_{1}, \ldots, C_{k}$ in $\operatorname{Num}(S)^{\prime}$.

Lemma 2.4. - $\operatorname{Aut}(S)$ acts faithfully on $\Lambda$, which is integral, even and unimodular of signature $(1,9)$, and spanned by the $U_{a b}$ and the

$$
f_{a b}=\frac{1}{2} F_{a b} .
$$

Remark 2.5. - Up to isometry there is a unique even unimodular lattice of signature $(1,9)$, often called $E_{10}$. So $\Lambda \cong E_{10}$. Lemma 2.4 is no surprise because if there are no new nodes then $S$ is an Enriques surface and $\Lambda$ coincides with $\operatorname{Num}(S)$, which is well-known to be a copy of $E_{10}$. Instead of $\Lambda$, it might seem simpler to

${ }^{(3)}$ Not to be confused with the Mukai lattice used in the context of moduli of sheaves on a K3 surface. 
consider the orthogonal complement of $C_{1}, \ldots, C_{k}$ in $\operatorname{Num}(S)$. This turns out to be inconvenient because the isometry type of the resulting lattice depends on the number of new nodes. (It is still $E_{10}$ if there is one new node, but not if there are more.) The Coble-Mukai lattice is the same in all cases.

Proof. - First we note that $\operatorname{Aut}(S)$ acts on $\Lambda$. This is because $\operatorname{Aut}(S)$ preserves the unique member of $\left|-2 K_{S}\right|$, namely $\mathcal{C}$. Therefore it permutes the components of $\mathcal{C}$. So it preserves $\operatorname{Num}(S)^{\prime}$ and their orthogonal complement in $\operatorname{Num}(S)^{\prime}$.

Next we prove $\operatorname{dim} \Lambda=10$. We remarked above that the $k=0$ case is a property of Enriques surfaces. So suppose $k>0$, in which case $S$ is rational. Then $\operatorname{dim}(\operatorname{Num}(S))=10-K_{S}^{2}$. (Blowing up a point increases both sides by 1 , while blowing down a $(-1)$-curve decreases both sides by 1 . So it is enough to check equality for $\mathbb{P}^{2}$.) Since $K_{S}^{2}=\frac{1}{4} \mathcal{C}^{2}=-k$ we get $\operatorname{dim}(\operatorname{Num}(S))=10+k$. Since $\mathcal{C}$ has $k$ components, we get $\operatorname{dim} \Lambda=10$.

Now we show that $\Lambda$ is integral. Any $x \in \Lambda$ may be expressed as $y+\sum_{i=1}^{k} r_{i} C_{i} / 2$ where $y \in \operatorname{Num}(S)$ and $r_{1}, \ldots, r_{k} \in \mathbb{Z}$. Rewriting this as $y=x-\sum r_{i} C_{i} / 2$, expressing $x^{\prime} \in \Lambda$ similarly, and using $\Lambda \perp C_{i}$ gives

$$
y \cdot y^{\prime}=x \cdot x^{\prime}+\sum r_{i} r_{i}^{\prime} \frac{C_{i} \cdot C_{i}}{4}=x \cdot x^{\prime}-\sum r_{i} r_{i}^{\prime} .
$$

This proves $x \cdot x^{\prime} \in \mathbb{Z}$.

Next we show that $\Lambda$ contains the $f_{a b}$. We saw in Lemma 2.3 that $\sigma$ acts nontrivially on the base $\mathbb{P}^{1}$ of the elliptic fibration $\left|\widetilde{G}_{a b}\right|$. Since an involution of $\mathbb{P}^{1}$ has exactly two fixed points, $\sigma$ sends exactly two fibers of this elliptic fibration to themselves. Choose one, call it $\widetilde{F}$, and write $\Pi$ for the corresponding plane in $\mathbb{P}^{3}$. Now, $\widetilde{F}$ is the total transform of the cubic plane curve residual to $L_{a b}$ in $\Pi$, and we write it as $\widetilde{F}=A+E_{1}+\cdots+E_{l}$. Here $E_{1}, \ldots, E_{l}$ are the $(-2)$-curves over the new nodes in $\Pi$ (if any), and $A$ is the sum of the proper transform of the plane cubic curve and the $(-2)$-curves over the vertices of the pentahedron that lie in the cubic plane curve (if any).

We will show that the corresponding fiber $F$ of $\left|G_{a b}\right|$ is something like a double fiber. Write $B$ for the image of $A$ in $S$, and choose the labeling so that $C_{1}, \ldots, C_{l} \subseteq S$ are the images of $E_{1}, \ldots, E_{l} \subseteq X$. Because $\sigma$ acts freely on $A$ away from $E_{1}, \ldots, E_{l}$, which it fixes pointwise, we have $F=2 B+C_{1}+\cdots+C_{l}$. That is, $f_{a b}=B+\frac{1}{2} C_{1}+\cdots+\frac{1}{2} C_{l}$, which lies in $\operatorname{Num}(S)^{\prime}$. It remains to show $f_{a b} \cdot C_{i}=0$ for all $i=1, \ldots, k$. Each $C_{i}$ lies in a fiber of $\left|G_{a b}\right|$, namely the one corresponding to the plane in $\mathbb{P}^{3}$ that contains $L_{a b}$ and the $i$ th new node. As a multiple of the class of a fiber, $f_{a b}$ pairs trivially with every component of every fiber.

Remark. - If $k=1$ then $E_{1}$ lies in one of the $\sigma$-invariant fibers, and we can choose $\widetilde{F}$ to be the other one. Then $f_{a b}=B$, so $f_{a b}$ lies in $\operatorname{Num}(S)$ not just $\operatorname{Num}(S)^{\prime}$. This leads to Remark 2.5's isomorphism, in the case of one new node, between $E_{10}$ and the orthogonal complement of $C_{1}$ in $\operatorname{Num}(S)$.

We continue the proof of Lemma 2.4. The $U_{a b}$ lie in $\Lambda$ and one can compute that their inner product matrix has signature $(1,9)$. So up to finite index they span $\Lambda$, which therefore also has this signature. One can check that the lattice spanned by 
the $U_{a b}$ and $f_{a b}$ is even unimodular. By integrality, $\Lambda$ can be no larger than this unimodular lattice. So we have proven all our claims except for the faithfulness of the action.

For this, suppose $g: S \rightarrow S$ is an isomorphism that preserves each $U_{a b}$. We know that $U_{01}$ meets each of $U_{23}, U_{34}, U_{24}$ in a single point. So each of these points is fixed. Since these points are distinct and $U_{01}$ is isomorphic to $\mathbb{P}^{1}$, we see that $g$ fixes $U_{01}$ pointwise. The same holds with other indices in place of 0,1 , so $g$ fixes every $U_{a b}$ pointwise.

Next, $g$ preserves the elliptic pencil $\left|G_{01}\right|$. Each of the curves $U_{23}, U_{24}, U_{34}$ has intersection number 2 with the class of the fiber, hence meets every fiber. So $g$ preserves every fiber. Furthermore, the restriction of $g$ to a general fiber preserves its intersection with $U_{23} \cup U_{24} \cup U_{34}$, so it has 6 fixed points. By [Sil09, Theorem III.10.1], every automorphism of an elliptic curve over a algebraically closed field of characteristic $\neq 2,3$ has order $1,2,3,4$ or 6 . (Fixing a single point means the automorphism preserves a Weierstrass equation, after which the result is easy.) In the last four cases, a Hurwitz formula calculation shows that $g$ has $4,3,2$ or 1 fixed points respectively. It follows that $g$ is the identity.

To each vertex $P_{a b}$ of the pentahedron is associated a birational involution $\widetilde{g}_{a b}$ of the Hessian surface. Namely, projection away from $P_{a b}$ defines a dominant rational map $H \rightarrow \mathbb{P}^{2}$ of degree 2. This realizes the function field of $H$ as a quadratic extension of that of $\mathbb{P}^{2}$, and $\widetilde{g}_{a b}$ is the nontrivial automorphism of this field extension. Again using the biregularity of birational maps between K3 surfaces, we regard the $\tilde{g}_{a b}$ as automorphisms of $X$.

Lemma 2.6. - Each $\widetilde{g}_{a b}$ commutes with the Cremona involution $\sigma$, hence descends to an automorphism of $S$.

Proof. - From the definition of $\widetilde{g}_{a b}$, it is enough to show, for a generic pair of points $u, v \in H$ with $u, v, P_{a b}$ collinear, that $\sigma(u), \sigma(v), P_{a b}$ are also collinear. Consider one of the 3 edges $L_{c d}$ of the pentahedron that pass through $P_{a b}$. By the definition of the $\left|\widetilde{G}_{c d}\right|$ in terms of planar sections of $H$, Lemma 2.3 says that the coplanarity of $u, v, L_{c d}$ implies that of $\sigma(u), \sigma(v), L_{c d}$. Applying this for each of the 3 edges shows that $\sigma(u), \sigma(v)$ lie on three planes, each containing one of these edges and in particular $P_{a b}$. The intersection of these planes is not a plane (since the 3 edges are not coplanar) and not a point (since it contains $\sigma(u), \sigma(v)$ ). Therefore it is a line, proving the collinearity of $\sigma(u), \sigma(v), P_{a b}$.

We write $g_{a b}$ for this induced automorphism of $S$. The next step is to examine how it acts on $\Lambda$. Its nature turns out to depend on whether the equality $\lambda_{a}=\lambda_{b}$ holds. Although we don't need it, we remark that $\lambda_{a}=\lambda_{b}$ if and only if $P_{a b}$ is an Eckardt point of the cubic surface $V_{3}$, meaning that 3 of the 27 lines on $V_{3}$ pass through $P_{a b}$; see [Dol12, Example 9.1.25]. To describe the $g_{a b}$ we must introduce some vectors $\alpha_{a b} \in \Lambda$ and some isometries $t_{a b}$ of $\Lambda$. We define

$$
\alpha_{a b}=f_{a b}-U_{a b},
$$


where $f_{a b}$ is from (2.4). One can check that $\alpha_{a b}$ has self-intersection -2 . For later use we record

$$
\begin{aligned}
& \alpha_{a b} \cdot U_{c d}= \begin{cases}2 & \text { if }\{a, b\}=\{c, d\} \\
0 & \text { otherwise }\end{cases} \\
& \alpha_{a b} \cdot \alpha_{c d}= \begin{cases}1 & \text { if }\{a, b\} \cap\{c, d\} \text { is a singleton, } \\
0 & \text { if }\{a, b\} \cap\{c, d\} \text { is empty. }\end{cases}
\end{aligned}
$$

The group $\mathfrak{S}_{5}$ of permutations of $0, \ldots, 4$ acts on $\Lambda$ by permuting the subscripts of the $U_{a b}$ and $f_{a b}$. We write $t_{a b}$ for the isometry of $\Lambda$ corresponding to the transposition $(a b)$.

Lemma 2.7 ([Dol18, Corollary 4.3]). - Suppose $\lambda_{a} \neq \lambda_{b}$. Then $g_{a b} \in \operatorname{Aut}(S)$ acts on $\Lambda \subset \operatorname{Num}(S)$ by the composition of $t_{a b}$ and the reflection in $\alpha_{a b}$.

Proof. - We write $\Pi_{a b} \subseteq \mathbb{P}^{3}$ for the plane spanned by $P_{a b}$ and $L_{a b}$. We claim that the projection involution $\widetilde{g}_{a b}$ sends $L_{a b}$ to neither itself nor $E_{a b}$. In fact, from $\lambda_{a} \neq \lambda_{b}$ it follows that $\Pi_{a b} \cap H$ consists of $L_{a b}$ and a plane cubic with a singularity at $P_{a b}$. By [Dol18, Lemma 4.6] this cubic is irreducible if and only if $\lambda_{a} \neq \lambda_{b}$. By definition, $\widetilde{g}_{a b}$ exchanges $L_{a b}$ and this cubic (or rather its proper transform).

To see how $g_{01}$ permutes the $U_{c d}$ other than $U_{01}$, we begin by working in the face $y_{3}=0$ of the pentahedron. This is the "front left" face in Figure 2.1, spanned by the topmost, bottommost and leftmost vertices. This face meets $H$ in 4 lines, two of which pass through $P_{01}$ and two of which do not, namely $L_{03}$ and $L_{13}$. For a generic line through $P_{01}$, by definition $\widetilde{g}_{01}$ swaps its two remaining intersection points with $H$. So $\widetilde{g}_{a b}$ swaps $L_{03}$ with $L_{13}$ and preserves their intersection point $P_{24}$, hence the divisor $E_{24}$ of $X$. Applying the same argument to the $y_{2}=0$ and $y_{4}=0$ faces shows that $\widetilde{g}_{01}$ exchanges $L_{0 c}$ with $L_{1 c}$ for each $c=2,3,4$, and preserves $E_{d e}$ for each pair $\{d, e\}$ disjoint from $\{0,1\}$.

Taking images under $X \rightarrow S$ shows that $g_{01}$ permutes the $U_{b c}$ other than $U_{01}$ by $t_{01}$. Their orthogonal complement is spanned by $\alpha_{01}$, which is therefore either fixed or negated by $g_{01}$. The first case leads to $g_{01}\left(U_{01}\right)=U_{01}$, which contradicts the first paragraph. So $g_{01}$ negates $\alpha_{01}$, completing our description of $g_{01}$ 's action on a basis for $\Lambda \otimes \mathbb{Q}$. It is obviously as stated in the Lemma 2.7. And similarly for the other involutions $g_{a b}$.

The reader may skip the rather technical second paragraph of the next Lemma 2.8. It is needed only for proving Theorem 4.3, which itself is not needed elsewhere in the paper.

Lemma 2.8. - Suppose $\lambda_{a}=\lambda_{b}$. Then $\widetilde{g}_{a b}$ acts on $H$ as the coordinate transposition $y_{a} \leftrightarrow y_{b}$, and $g_{a b}$ acts on $\Lambda \subset \operatorname{Num}(S)$ as $t_{a b}$. Furthermore, $\alpha_{a b} \in \Lambda$ is represented by a rationally effective divisor.

More specifically, if the plane $\Pi_{a b} \subset \mathbb{P}^{3}$ spanned by $L_{a b}$ and $P_{a b}$ contains no new node, then $\alpha_{a b}$ is the class of a $(-2)$-curve. Otherwise $\Pi_{a b}$ contains exactly two new nodes and $\alpha_{a b}=2[D]+\frac{1}{2}\left[C_{i}\right]+\frac{1}{2}\left[C_{j}\right]$, where $D$ is a $(-1)$-curve and $C_{i}, C_{j}$ are the $(-4)$-curves corresponding to the two new nodes.

Proof. - Suppose $x$ is a point of $H$, and let $x^{\prime}$ be its image under the coordinate transposition $(a b)$. Clearly it lies in $H$. Also, $x, x^{\prime}$ and $P_{a b}$ are collinear. For $x$ generic, 
this shows that the definition of $\widetilde{g}_{a b}$ is to swap $x$ with $x^{\prime}$. This proves our claims about $\widetilde{g}_{a b}$ and $g_{a b}$.

Under the hypothesis $\lambda_{a}=\lambda_{b}$, we get $\Pi_{a b} \cap H=2 L_{a b}+M+M^{\prime}$ where $M, M^{\prime}$ are lines through $P_{a b}$ (possibly coincident). We keep the same notation $M, M^{\prime}$ for their proper transforms in $X$. The fiber $\widetilde{F}$ of $\left|\widetilde{G}_{a b}\right|$ corresponding to $\Pi_{a b}$ is

$$
\widetilde{F}=L_{a b}+M+M^{\prime}+E_{a b}+E_{1}+\cdots+E_{l}
$$

where $E_{1}, \ldots, E_{l}$ are the exceptional divisors over the new nodes that lie in $\Pi_{a b}$ (if any). Because $\sigma$ exchanges $L_{a b}$ with $E_{a b}$, it preserves this fiber. Arguing as in the proof of Lemma 2.4 (with $A=L_{a b}+M+M^{\prime}+E_{a b}$ ) shows that

$$
f_{a b}=B+\frac{1}{2} C_{1}+\cdots+\frac{1}{2} C_{l}
$$

where $B=U_{a b}+D$ with $D$ an effective divisor. This shows that $\alpha_{a b}=f_{a b}-U_{a b}$ is rationally effective.

A more detailed analysis leads to two cases. If $\Pi_{a b}$ contains no new nodes, then $\sigma$ must act freely on $M \cup M^{\prime}$. This forces $M, M^{\prime}$ to be distinct. So $\widetilde{F}$ is a cycle of four $(-2)$-curves. Taking the quotient by $\sigma$ shows that $f_{a b}$ is the sum of two $(-2)$-curves intersecting each other twice. One is $U_{a b}$ and $D$ is the other.

On the other hand, if $\Pi_{a b}$ contains a new node, then the planar section $M+M^{\prime}+L_{a b}$ of $H$ contains that node with multiplicity two. So the node lies on both $M$ and $M^{\prime}$. Since $P_{a b}$ also lies on these lines, we get $M=M^{\prime}$. Since $\sigma$ has exactly two fixed points on $M, \Pi_{a b}$ contains exactly two new nodes. So $\widetilde{F}=L_{a b}+2 M+E_{a b}+E_{i}+E_{j}$ where $E_{i}, E_{j}$ are the $(-2)$-curves in $X$ lying over these new nodes. So $f_{a b}=U_{a b}+$ $2 D+\frac{1}{2} C_{i}+\frac{1}{2} C_{j}$, where $D$ is the image of $M$ in $S$. Since $D$ is clearly a smooth curve $D$ that meets $U_{a b}, C_{i}, C_{j}$ once each, the relation $f_{a b}^{2}=0$ forces $D^{2}=-1$.

\section{The nef cone}

We continue in the situation of Section 2. In particular, $\lambda_{0}, \ldots, \lambda_{4} \neq 0$ are the parameters of the cubic surface in Sylvester pentahedral form, and $S$ is the Enriques or Coble surface obtained from its Hessian. We defined the Coble-Mukai lattice as a certain 10-dimensional lattice $\Lambda \subset \operatorname{Num}_{\mathbb{Q}}(S)$, and showed that $\operatorname{Aut}(S)$ acts faithfully on it. The main object in this section is the intersection of the real nef cone $\operatorname{Nef}_{\mathbb{R}}(S)$ with $\Lambda_{\mathbb{R}}=\Lambda \otimes \mathbb{R}$. In the next section we will use this to compute $\operatorname{Aut}(S)$ in the special case $\left(\lambda_{0}, \ldots, \lambda_{4}\right)=(1,1,1,1, t)$. But we impose no condition on the $\lambda_{a}$ in this section.

The signature of $\Lambda$ is $(1,9)$, so the set of positive-norm lines in $\Lambda_{\mathbb{R}}$ forms a copy of hyperbolic 9-space $H^{9}$. In Section 2 we defined twenty vectors $U_{a b}, \alpha_{a b} \in \Lambda$. We define

$$
P=\left\{x \in \Lambda_{\mathbb{R}} \mid x \cdot U_{a b} \geqslant 0 \text { and } x \cdot \alpha_{a b} \geqslant 0 \text { for all } a, b\right\} .
$$

We will usually pass freely between $P$ and the corresponding hyperbolic polytope. One can work out the dihedral angles of $P$ from the fact that the $U_{a b}$ and $\alpha_{a b}$ have norm -2 and their pairwise inner products are 0,1 or 2 by (2.6)-(2.7). Namely, two facets meet orthogonally when the inner product is 0 , and make angle $\pi / 3$ when the 
inner product is 1 . When the inner product is 2 , the facets are parallel in the sense that the hyperplanes containing them meet at the boundary of $H^{9}$ but not in $H^{9}$ itself. The language comes from the corresponding example in the upper half-plane model of $H^{2}$ : two vertical half-lines.

This shows that $P$ is a Coxeter polytope, meaning that if two facets meet then their dihedral angle is an integral submultiple of $\pi$. Such polytopes have a long history in the theory of algebraic surfaces, for example $P$ itself is the nef cone of the Enriques surface $K_{V I}$ of Type VI in Kondo's classification of complex Enriques surfaces with finite automorphism group [Kon86]. We will use the following background material about Coxeter polytopes; for more information see [Dol08, Vin71].

A Coxeter polytope is usually understood in terms of its Coxeter diagram, which is a graph with one vertex for each facet of the polytope, and edges that indicate the dihedral angles between facets. We use Kondo's conventions that orthogonality corresponds to the absence of an edge, a dihedral angle $\pi / 3$ corresponds to a single edge, and parallelism corresponds to a double edge. (Caution: double edges do not have the same meaning as in Lie theory, where they indicate a dihedral angle $\pi / 4$.)

The Coxeter diagram $D$ of $P$ is rather complicated, but can be described as follows. The facets corresponding to the $U_{a b}$ form a copy of the Petersen graph (Figure 2.2). The facets corresponding to the $\alpha_{a b}$ form a copy of the "anti-Petersen" graph, meaning that $\alpha_{a b}$ and $\alpha_{c d}$ are joined just if $U_{a b}$ and $U_{c d}$ are not. Finally, each $U_{a b}$ is joined to $\alpha_{a b}$ by a double edge. A drawing of this graph, due to Kondo [Kon86], appears in Figure 3.1. Calculations like Lemma 4.1 below are usually easiest using the Petersen/anti-Petersen description rather than the figure, because the $\mathfrak{S}_{5}$ symmetry is manifest.

For each facet of a Coxeter polytope, one chooses an outward-pointing normal vector and calls it the simple root of that facet. In some situations there is a question of how to normalize the simple roots, but we always take vectors of norm -2 . For example, the $\alpha_{a b}$ and $U_{a b}$ are the simple roots of $P$. The center of $P$ is represented by $\Delta=\sum_{\{a, b\}} U_{a b}$, which has inner product 1 with each $U_{a b}$, inner product 2 with each $\alpha_{a b}$, and norm 10 .

Kondo showed that $P$ (regarded as a hyperbolic polytope) has finite volume [Kon86, p. 226]; actually he proved the equivalent statement that the Coxeter group of $P$ has finite index in Aut $\Lambda$.

Remark 3.1. - We mentioned that $P$ is the nef cone of the Enriques surface $K_{V I}$. Its facets correspond to $(-2)$-curves on $K_{V I}$, and Figure 3.1 is the dual graph of these curves. This particular surface $K_{V I}$ has Hessian type, coming from the construction of Section 2 applied to the Clebsch diagonal surface. That is, with $\left(\lambda_{0}, \ldots, \lambda_{4}\right)=(1,1,1,1,1)$.

The polytope $P$ also occurs in Shimada's paper [Shi19], where his Theorem 1.8 shows that $P$ is the union of $2^{14} \cdot 3 \cdot 5 \cdot 7 \cdot 17 \cdot 31$ fundamental chambers for the full reflection group $W_{237}$ of the lattice $\Lambda \cong E_{10}$.

For any Enriques surface $S$ of Sylvester-nondegenerate Hessian type, the class $\Delta \in \operatorname{Pic}(S)$ represents an ample Fano polarization on $S$ that realizes the surface as a surface of degree 10 in $\mathbb{P}^{5}$, the smallest possible projective embedding of an Enriques surface (see, for example, [Dol16, p. 14]). Each such ample polarization, 


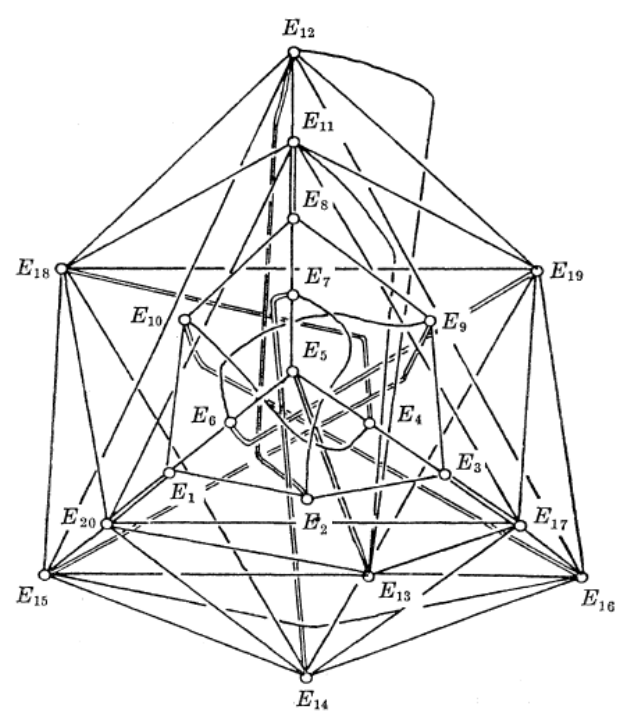

$\begin{array}{cccccccccc}U_{01} & U_{02} & U_{03} & U_{04} & U_{12} & U_{13} & U_{14} & U_{23} & U_{24} & U_{34} \\ E_{1} & E_{8} & E_{4} & E_{7} & E_{5} & E_{9} & E_{10} & E_{2} & E_{3} & E_{6} \\ \alpha_{01} & \alpha_{02} & \alpha_{03} & \alpha_{04} & \alpha_{12} & \alpha_{13} & \alpha_{14} & \alpha_{23} & \alpha_{24} & \alpha_{34} \\ E_{20} & E_{11} & E_{18} & E_{14} & E_{13} & E_{15} & E_{16} & E_{12} & E_{17} & E_{19}\end{array}$

Figure 3.1. Kondo's drawing of the Coxeter diagram of the polytope $P$, and how his labels correspond to ours.

taken with multiplicity 3 , is equal to the sum of ten isotropic nef divisors (in our case, the $f_{a b}$ ), all of whose pairwise intersection numbers are 1 . Such a polarization (maybe quasi-ample instead of ample) exists on any Enriques surface. In our case, the associated projective embedding sends each $U_{a b}$ to a line and any divisor representing $f_{\alpha}$ to a plane cubic curve.

Theorem 3.2. - Let $G_{0}$ be the subgroup of Aut $(\Lambda)$ generated by those $g_{c d}$ for which $\lambda_{c} \neq \lambda_{d}$. Then $\operatorname{Nef}_{\mathbb{R}}(S) \cap \Lambda_{\mathbb{R}}$ is the closure $Q$ of the union of the $G_{0}$-images of the polytope $P$. Furthermore, the facets of $Q$ correspond to the $G_{0}$-images of the $U_{a b}$ and of those $\alpha_{a b}$ for which $\lambda_{a}=\lambda_{b}$.

Proof. - The $U_{a b}$ are effective divisors, and the $\alpha_{a b}$ with $\lambda_{a}=\lambda_{b}$ are rationally effective by Lemma 2.8. We call the corresponding facets of $P$ the exterior facets. Each remaining facet of $P$ corresponds to some $\alpha_{c d}$ with $\lambda_{c} \neq \lambda_{d}$. Lemma 2.7 shows that this facet is also a facet of $g_{c d}(P)$, which lies on the other side of the facet. It follows that the boundary of $Q$ in $H^{9}$ consists of the $G_{0}$-images of the exterior facets of $P$. This proves the last claim of the Theorem 3.2, and shows that every facet of $Q$ is orthogonal to a rationally effective divisor. This $\operatorname{implies} \operatorname{Nef}_{\mathbb{R}}(S) \cap \Lambda_{\mathbb{R}} \subseteq Q$.

Suppose that the inclusion $\operatorname{Nef}_{\mathbb{R}}(S) \cap \Lambda_{\mathbb{R}} \subseteq Q$ were strict. By definition, $\operatorname{Nef}_{\mathbb{R}}(S)$ is the intersection of the half-spaces $x \cdot B \geqslant 0$ in $\operatorname{Num}_{\mathbb{R}}(S)$, where $B$ varies over the effective divisors on $S$. So $S$ has some effective divisor $B$, whose orthogonal complement in $\operatorname{Num}_{\mathbb{R}}(S)$ meets the interior of $Q$. Write $\beta$ for the projection of its class in $\operatorname{Num}(S)$ to $\Lambda$. Without loss of generality we replace $B$ by its $G_{0}$-image 
having smallest possible $\beta \cdot \Delta$. This corresponds to the hyperplane $\beta^{\perp}$ coming as close as possible to $\Delta$ in hyperbolic space.

We claim that $\beta \cdot \alpha_{c d} \geqslant 0$ when $\lambda_{c} \neq \lambda_{d}$. Supposing to the contrary that $\beta \cdot \alpha_{c d}<0$, we write $\beta^{\prime}=\beta+\left(\beta \cdot \alpha_{c d}\right) \alpha_{c d}$ for the image of $\beta$ under the reflection in $\alpha_{c d}$. Then we compute

$$
\begin{aligned}
g_{c d}(\beta) \cdot \Delta & =t_{c d}\left(\beta^{\prime}\right) \cdot \Delta=\beta^{\prime} \cdot t_{c d}^{-1}(\Delta)=\beta^{\prime} \cdot \Delta \\
& =\left(\beta+\left(\beta \cdot \alpha_{c d}\right) \alpha_{c d}\right) \cdot \Delta=\beta \cdot \Delta+2 \beta \cdot \alpha_{c d}<\beta \cdot \Delta
\end{aligned}
$$

where we have used the description of $g_{a b}$ from Lemma 2.7, the invariance of $\Delta$ under $t_{c d} \in \mathfrak{S}_{5}$, and $\alpha_{c d} \cdot \Delta=2$. This contradicts the choice of $B$.

Because $U_{a b} \in \Lambda$ we also have $\beta \cdot U_{a b}=B \cdot U_{a b} \geqslant 0$ for all $a, b$. When $\lambda_{a}=\lambda_{b}$, the same argument gives $\beta \cdot \alpha_{a b}=B \cdot \alpha_{a b} \geqslant 0$ because $\alpha_{a b}$ is rationally effective. We have shown that $\beta$ has nonnegative inner products with the simple roots of $P$. That is, it lies in $P$. Regarded as a hyperbolic polytope, $P$ has finite volume. Therefore, regarded as a cone in $\Lambda_{\mathbb{R}}$, it consists of norm $\geqslant 0$ vectors. This contradicts the hypothesis that $\beta^{\perp}$ meets the interior of $Q$ (or indeed any point of hyperbolic space).

\section{Automorphism groups}

Our goal in this section is to work out the automorphism groups of the Enriques and Coble surfaces $S$ constructed in Section 2, for the parameters $\left(\lambda_{0}, \ldots, \lambda_{4}\right)=$ $(1,1,1,1, t)$, with $t \neq 0$ as always. By Lemma 2.4 , Aut $(S)$ acts faithfully on the lattice $\Lambda \subset \operatorname{Num}_{\mathbb{Q}}(S)$. And Theorem 3.2 describes the invariant cone $Q=\operatorname{Nef}_{\mathbb{R}}(S) \cap \Lambda_{\mathbb{R}}$ fairly explicitly. The idea in this section is to use the shape of this cone to show that $\operatorname{Aut}(S)$ is generated by the known automorphisms $g_{a b}$.

In the particular case $t=1$, the cubic surface is the Clebsch diagonal cubic surface. It is smooth except in characteristic 5 , in which case it has an ordinary node at $(1: 1: 1: 1: 1)$. Regardless of whether there is a node, Theorem 3.2 shows that $Q=P$. The isometry group of $P$ is the automorphism group of its Coxeter diagram, which is just the obvious group $\mathfrak{S}_{5}$ permuting the pentahedral coordinates $y_{0}, \ldots, y_{4}$. So $\operatorname{Aut}(S)=\mathfrak{S}_{5}$. Over $\mathbb{C}$, this Enriques surface has type VI in Kondo's classification of complex Enriques surfaces with finite automorphism group; see Remark 3.1.

We remark on the other special surfaces in the family. If $t \notin\left\{\frac{1}{16}, \frac{1}{4}\right\}$ then the Hessian surface $H$ is smooth away from the vertices of the Sylvester pentahedron. In this case the Cremona involution $\sigma$ acts freely on $X$, so $S$ is an Enriques surface. When $t=\frac{1}{16}$ there is one new node, at $(1: 1: 1: 1:-4)$. When $t=\frac{1}{4}$ there are four new nodes, at the images of $(1: 1: 1:-1:-2)$ under the permutations of the first four coordinates.

A cusp (or ideal vertex) of a Coxeter polytope in $H^{9}$ means a point of the boundary $\partial H^{9}$, such that the simple roots orthogonal to it span a rank 9 subspace of $\mathbb{R}^{1,9}$. The proof of Theorem 4.2 below relies on a comparison between the cusps of $P$ and those of $Q$. Cusps can be recognized easily from the Coxeter diagram, in terms of parabolic subdiagrams (also called affine). This means a subset of vertices, together with all 
edges between them, which is a disjoint union of copies of the standard extended Dynkin diagrams $\widetilde{A}_{n}, \widetilde{D}_{n}, \widetilde{E}_{n}$. The rank of the subdiagram means the sum of the subscripts, and cusps correspond bijectively to parabolic subdiagrams of rank 8 . The idea behind this bijection is that the simple roots corresponding to a parabolic subdiagram have negative semidefinite inner product matrix, and the nullspace of their span determines a point of $\partial H^{9}$. And on the other hand, in the upper half space model, with a cusp at infinity, the intersection of the Coxeter polytope with a horizontal plane is a Euclidean Coxeter polytope. And such polytopes are classified by parabolic diagrams. The diagrams $\widetilde{B}_{n}, \widetilde{C}_{n}, \widetilde{F}_{4}, \widetilde{G}_{2}$ do not occur for us because they involve dihedral angles $\pi / 4$ or $\pi / 6$. See [Vin72, Sections 2-3] or [Dol08] for more background.

Lemma 4.1. - There are four $\mathfrak{S}_{5}$-orbits of cusps of $P$, corresponding to subdiagrams $\widetilde{A}_{5} \widetilde{A}_{1} \widetilde{A}_{2}, \widetilde{E}_{6} \widetilde{A}_{2}, \widetilde{D}_{5} \widetilde{A}_{3}$ and $\widetilde{A}_{4} \widetilde{A}_{4}$ of $D$. In each case the first listed component lies in the Petersen graph rather than the anti-Petersen graph, hence corresponds to some set of divisors $U_{a b}$.

Proof. - It is not hard to directly classify the connected parabolic diagrams $K$ in $D$ up to the action of $\mathfrak{S}_{5}$. It turns out that the vertices of $D$ not joined to $K$ form a parabolic diagram, which together with $K$ has rank 8 . Kondo had to perform this computation in his proof that $P$ has finite volume, so one can rely on his results recorded in [Kon86, p. 226].

Theorem 4.2. - Let $S$ be the Enriques or Coble surface arising from the Hessian of a Sylvester non-degenerate cubic surface with parameters

$$
\left(\lambda_{0}, \ldots, \lambda_{4}\right)=(1,1,1,1, t) .
$$

Then $\operatorname{Aut}(S)$ is generated by the ten involutions $g_{a b}$.

Proof. - We treated the $t=1$ case above, so we suppose $t \neq 1$. Theorem 3.2 shows that $Q$ is (the closure of) the union of the $G_{0}$-translates of $P$. Here $G_{0}$ is the subgroup of $\operatorname{Aut}(S)$ generated by those $g_{c d}$ with $\lambda_{c} \neq \lambda_{d}$. We will also write $G$ for the group generated by all ten $g_{a b}$. Suppose $g \in \operatorname{Aut}(S)$. Our strategy is to replace $g$ by its compositions with elements of $G$, ultimately leading to the conclusion that $g$ is the identity. The main idea of the proof is to show that the tessellation of $Q$ by copies of $P$ is intrinsic, so that $g$ preserves it. We will do this by comparing the cusps of $Q$ to the cusps of $P$. As in the proof of Theorem 3.2 we use the term "exterior facets" for the facets of $P$ corresponding to the $U_{a b}$ and to those $\alpha_{a b}$ with $\lambda_{a}=\lambda_{b}$. We call the other facets "interior".

The fact that $\lambda_{4}$ is different from $\lambda_{0}, \ldots, \lambda_{3}$ breaks the $\mathfrak{S}_{5}$ symmetry. Each $\mathfrak{S}_{5^{-}}$ orbit of cusps of $P$ breaks up into several orbits under the subgroup $\mathfrak{S}_{4}$ acting on the indices $\{0,1,2,3\}$. We will focus on the cusps of type $\widetilde{E}_{6} \widetilde{A}_{2}$. There is one such cusp for every ordered pair $a, b$ of distinct elements of $\{0, \ldots, 4\}$. Namely, $\widetilde{E}_{6}(a, b)$ has $U_{a b}$ as the branch node, $U_{c d}, U_{d e}, U_{e c}$ as its neighbors, and $U_{a e}, U_{a c}, U_{a d}$ as the end nodes. Here $\{c, d, e\}=\{0, \ldots, 4\}-\{a, b\}$. The nodes of $D$ not joined to $\widetilde{E}_{6}(a, b)$ are $\alpha_{b c}, \alpha_{b d}, \alpha_{b e}$, which form an $\widetilde{A}_{2}$ diagram. The corresponding facets of $P$ can be either exterior or interior. Under $\mathfrak{S}_{4}$ there are three orbits of ordered pairs of distinct 
elements of $\{0, \ldots, 4\}$, so there are three orbits of $\widetilde{E}_{6} \widetilde{A}_{2}$ diagrams, represented by

$$
\begin{array}{ccc} 
& \text { exterior } \widetilde{A}_{2} \text { facets } & \text { interior } \widetilde{A}_{2} \text { facets } \\
\widetilde{E}_{6}(4,0) & \alpha_{01}, \alpha_{02}, \alpha_{03} & \text { none } \\
\widetilde{E}_{6}(0,1) & \alpha_{12}, \alpha_{13} & \alpha_{14} \\
\widetilde{E}_{6}(0,4) & \text { none } & \alpha_{14}, \alpha_{24}, \alpha_{34}
\end{array}
$$

It is easy to write down a vector in $\Lambda$ representing the cusp corresponding to a given $\widetilde{E}_{6}(a, b)$, namely the null vector

$$
\nu_{a, b}=3 U_{a b}+2\left(U_{c d}+U_{d e}+U_{e c}\right)+\left(U_{a e}+U_{a c}+U_{a d}\right) .
$$

(This comes from Lie theory. The lowest root of the $E_{6}$ root system extends the $E_{6}$ Dynkin diagram to $\widetilde{E}_{6}$. Our formula, modulo $\nu_{a, b}$, is the standard linear dependence between it and the simple roots of $E_{6}$.)

Set $\nu=\nu_{4,0}$. Since all the facets of $P$ incident to $\nu$ are exterior facets, $\nu$ is also a cusp of $Q$. Since $g(\nu)$ is a cusp of $Q$, it is a cusp of some $G_{0^{-}}$translate of $P$. By replacing $g$ by its composition with a suitable element of $G_{0}$, we may therefore suppose without loss that $g(\nu)$ is a cusp of $P$. The facets of $Q$ containing $g(\nu)$ might not coincide with the facets of $P$ containing $g(\nu)$, because $Q$ might contain several translates of $P$ that are incident to $g(\nu)$. Nevertheless, every exterior facet of $P$ that contains $g(\nu)$ will also be a facet of $Q$. Lemma 4.1 shows that these facets of $P$ account for a subdiagram $\widetilde{A}_{5}, \widetilde{E}_{6}, \widetilde{D}_{5}$ or $\widetilde{A}_{4}$ of $g(\nu)$ 's diagram in $Q$. The cases $\widetilde{A}_{5}$, $\widetilde{D}_{5}$ and $\widetilde{A}_{4}$ are incompatible with the fact that the diagram of $g(\nu)$ in $Q$ is $\widetilde{E}_{6} \widetilde{A}_{2}$. It follows that the $\widetilde{E}_{6}$ component, of the diagram of $g(\nu)$ as a cusp of $Q$, consists of exterior walls of $P$. By replacing $g$ by its composition with some element of $\mathfrak{S}_{4} \subset G$, we may suppose without loss that $g(\nu)$ is one of the cusps $\nu_{4,0}, \nu_{0,1}$ or $\nu_{0,4}$ of $P$.

We claim that the first of these three cases holds, which is to say that $g$ fixes the cusp $\nu_{4,0}$. One can check that $g_{14}$ exchanges the other two cusps, by using the explicit formula (4.1) for vectors representing them. So in the case $g(\nu)=\nu_{0,1}$ we may replace $g$ by its composition with $g_{14}$, reducing to the case $g(\nu)=\nu_{0,4}$. In this case we will derive a contradiction. The facets $\alpha_{14}, \alpha_{24}, \alpha_{34}$ of $P$ are incident to $\nu_{0,4}$, but they are interior facets. Therefore $Q$ also contains the images of $P$ under $g_{14}, g_{24}, g_{34}$. We will focus on $g_{14}(P)$. We already noted that $\alpha_{12}$ is an exterior facet of $P$ incident to $\nu_{0,1}$, and that $g_{14}$ sends $\nu_{0,1}$ to $\nu_{4,0}$. Therefore $g_{14}\left(\alpha_{12}\right)=\alpha_{14}+\alpha_{24}$ is a facet of $Q$ incident to $\nu_{0,4}$. Since $\nu_{0,4}$ is invariant under permutations of the indices $1,2,3$, the remaining two facets of $Q$ at $\nu_{0,4}$ are $\alpha_{24}+\alpha_{34}$ and $\alpha_{34}+\alpha_{14}$.

In summary, the facets of $Q$ at $\nu_{4,0}$ are $\widetilde{E}_{6}(4,0)$ and $\alpha_{01}, \alpha_{02}, \alpha_{03}$, while the facets of $Q$ at $\nu_{0,4}$ are $\widetilde{E}_{6}(0,4)$ and $\alpha_{14}+\alpha_{24}, \alpha_{24}+\alpha_{34}, \alpha_{34}+\alpha_{14}$. Under our assumption $g\left(\nu_{4,0}\right)=\nu_{0,4}$, we see that $g$ sends $\widetilde{E}_{6}(0,4)$ to $\widetilde{E}_{6}(4,0)$ and $\left\{\alpha_{01}, \alpha_{02}, \alpha_{03}\right\}$ to $\left\{\alpha_{14}+\right.$ $\left.\alpha_{24}, \alpha_{24}+\alpha_{34}, \alpha_{34}+\alpha_{14}\right\}$. This contradicts

$$
\begin{aligned}
\alpha_{01}+\alpha_{02}+\alpha_{03} & =\frac{1}{2} \nu_{4,0} \\
\left(\alpha_{14}+\alpha_{24}\right)+\left(\alpha_{24}+\alpha_{34}\right)+\left(\alpha_{34}+\alpha_{14}\right) & =\nu_{0,4} .
\end{aligned}
$$

(The fact that the right side is twice as large in the second line is the numerical manifestation of the idea that the cusp of $Q$ at $\nu_{0,4}$ is "twice as big in the $A_{2}$ direction" 
as the cusp of $Q$ at $\nu_{4,0}$. One should visualize an equilateral triangle of edge length 2 , divided into four equilateral triangles of edge length 1.)

We have reduced to the case that $g$ fixes $\nu_{4,0}$. The center of the polytope $P$ is represented by $\Delta=\sum_{\{a, b\}} U_{a b}$. It has inner product 1 with each $U_{a b}$, inner product 2 with each $\alpha_{a b}$, and inner product 10 with itself. We may characterize it in terms of the cusp $\nu_{4,0}$ as follows. It is the unique norm 10 element of $\Lambda$ that is effective, has inner product 1 with each root of the $\widetilde{E}_{6}$ diagram at $\nu_{4,0}$, and has inner product 2 with each root of the $\widetilde{A}_{2}$ diagram there. Therefore $g$ preserves $\Delta$.

If $r \in \Lambda$ is the class of a $(-2)$ curve, then $r \cdot \Delta \geqslant 1$, with equality if and only if $r$ equals some $U_{a b}$. So $g$ permutes the $U_{a b}$, hence the $\alpha_{a b}$, hence preserves $P$. Its action on $P$ is determined by its action on the Coxeter diagram $D$. Since $g$ preserves $Q$, it permutes the interior facets $\alpha_{a 4}$ of $P$ among themselves. By replacing $g$ by its composition with an element of $\mathfrak{S}_{4} \subset G$, we may suppose that it preserves each of them. The only automorphism of the Coxeter diagram $D$ with this property is the identity. So $g$ acts by the identity on $\Lambda$. Then Lemma 2.4 shows that $g$ is the identity, completing the proof.

The following result is not needed elsewhere in the paper.

TheOREM 4.3. - For $t \neq \frac{1}{4}$, there are two orbits of $\operatorname{Aut}(S)$ on the set of $(-2)$ curves, with orbit representatives $U_{01}, \alpha_{01}$. For $t=\frac{1}{4}$, no $\alpha_{a b}$ is represented by a $(-2)$-curve, and every $(-2)$-curve is $\operatorname{Aut}(S)$-equivalent to $U_{01}$.

Proof. - Consider the simple roots of $Q$. By Theorem 3.2 these are the Aut $(S)$ images of the $U_{a b}$ and of those $\alpha_{c d}$ for which $\lambda_{c}=\lambda_{d}$. Each $U_{a b}$ is a (-2)-curve. And by Lemma 2.8, each of these $\alpha_{c d}$ is either represented by a (-2)-curve, or else has the form $\alpha_{c d}=2[D]+\frac{1}{2}\left[C_{i}\right]+\frac{1}{2}\left[C_{j}\right]$ where $D$ is a $(-1)$-curve and $C_{i}, C_{j}$ are two components of the bi-anticanonical divisor (and in particular are $(-4)$-curves). The latter case occurs if and only if the plane $\Pi_{c d} \subset \mathbb{P}^{3}$ containing $L_{c d}$ and $P_{c d}$ contains two new nodes. This happens only when $t=\frac{1}{4}$, and then it happens for all $c, d \neq 4$.

Now suppose $R$ is a (-2)-curve in $S$. The adjunction formula forces $R \cdot K_{S}=0$, so $R$ misses the components $C_{1}, \ldots, C_{k}$ of the bi-anticanonical divisor. It follows that the class of $R$ in $\operatorname{Num}(S)$ lies in $\Lambda$. We claim that $R$ equals one of the $(-2)$-curves from the previous paragraph. Otherwise it would have inner product $\geqslant 0$ with all the simple roots of $Q$. But these define (the intersection with $\Lambda_{\mathbb{R}}$ of) the nef cone, so $R$ would be nef, contrary to $R^{2}<0$. Therefore either $R$ is an $\operatorname{Aut}(S)$-image of some $U_{a b}$, or else $t \neq \frac{1}{4}$ and $R$ is an $\operatorname{Aut}(S)$-image of some $\alpha_{c d}$ with $\lambda_{c}=\lambda_{d}$.

When $t=1$ we have seen that $\operatorname{Aut}(S)=\mathfrak{S}_{5}$, whose orbits on the simple roots of $Q=P$ have representatives $U_{01}, \alpha_{01}$. So suppose $t \neq 1$. Using $\mathfrak{S}_{4} \subset \operatorname{Aut}(S)$ shows that $R$ is $\operatorname{Aut}(S)$-equivalent to $U_{01}, U_{04}$ or $\alpha_{01}$, with the last case only possible when $t \neq \frac{1}{4}$. We claim $g_{14}\left(U_{04}\right)=U_{01}$. To see this, recall that $g_{14}$ acts by the transposition (14) on subscripts, followed by reflection in $\alpha_{14}$. The transposition sends $U_{04}$ to $U_{01}$, which is orthogonal to $\alpha_{14}$. This proves our claim.

All that remains to prove is that $U_{01}$ and $\alpha_{01}$ are not $\operatorname{Aut}(S)$-equivalent. It suffices to show that they are not equivalent under the much larger group $W \rtimes \operatorname{Aut}(P)$, where $W$ is the Coxeter group of the polytope $P$. This is equivalent to the nonconjugacy in $W \rtimes \operatorname{Aut}(P)$ of the reflections of $\Lambda$ corresponding to $U_{01}$ and $\alpha_{01}$. 
To prove this non-conjugacy we construct a homomorphism $W \rtimes \operatorname{Aut}(P) \rightarrow(\mathbb{Z} / 2)^{2}$ that sends the reflections of $U_{01}$ and $\alpha_{01}$ to distinct elements of $(\mathbb{Z} / 2)^{2}$. We will need the standard presentation of $W$, which is as follows [Bou81, Chapter V, Section 4], [Vin71, Theorem 2]. It has one generator for each vertex of $D$, namely the reflection across the corresponding facet of $P$. These have order 2 , two of them commute if their vertices unjoined (because the facets are orthogonal), and two of them have product of order 3 if their vertices are joined by a single edge (because the dihedral angle is $\pi / 3$ ). These relations define $W$. The action of $\mathfrak{S}_{5}$ on $W$ is by permuting the generators in the way that it permutes the facets of $P$.

Our homomorphism sends the reflections in the $U_{a b}$ to $(1,0)$, the reflections in the $\alpha_{a b}$ to $(0,1)$, and $\mathfrak{S}_{5}$ to the trivial element. It is easy to check that all the relations are satisfied, so this does in fact define a homomorphism.

TheOrem 4.4. - Suppose $S$ is as in Theorem 4.2, with $t \neq 1$. Then

$$
\operatorname{Aut}(S)=((\mathbb{Z} / 2) *(\mathbb{Z} / 2) *(\mathbb{Z} / 2) *(\mathbb{Z} / 2)) \rtimes \mathfrak{S}_{4}
$$

where the factors of the free product are generated by $g_{04}, g_{14}, g_{24}, g_{34}$. Also, the group $\mathfrak{S}_{4}$ is generated by the $g_{a b}$ with $a, b \neq 4$, and permutes the factors of the free product in the obvious way.

Proof. - Because the action of $\operatorname{Aut}(S)$ on $\Lambda$ is faithful and independent of the parameter $t$ and the characteristic $(\neq 2,3)$ of $\mathbb{k}$, it suffices to prove this for $t=\frac{1}{16}$ in characteristic 0 . In this case $k=1$, so the unique bi-anticanonical divisor of $S$ is a smooth rational curve. This yields a homomorphism $\operatorname{Aut}(S) \rightarrow \mathrm{PGL}_{2}(\mathbb{k})$. We show in Theorem 5.7 that the images $\bar{g}_{a 4}$ of the $g_{a 4}$ generate the factors of a free product $(\mathbb{Z} / 2)^{* 4} \subset \mathrm{PGL}_{2}(\mathbb{k})$. It follows that the same holds for the $g_{a 4}$ as elements of $\operatorname{Aut}(S)$. The rest of the Theorem 4.4 is obvious.

Remark 4.5. - Theorem 4.4 is an analogue of I. Shimada's calculation of $\operatorname{Aut}(S)$ when $S$ is the Enriques surface arising from a general Sylvester non-degenerate cubic surface [Shi19, Theorem 1.2]. Shimada used Borcherds' method, which is more technical but similar in flavor to ours. He showed that $\operatorname{Aut}(S)$ is generated by the $g_{a b}$, with defining relations

$$
g_{a b}^{2}=1,\left(g_{a b} g_{b c} g_{c a}\right)^{2}=1,\left(g_{a b} g_{c d}\right)^{2}=1
$$

where $\{a, b\} \cap\{c, d\}=\emptyset$ in the last relation. It is an interesting question whether our methods could be adapted to recover his result, and possibly even compute $\operatorname{Aut}(S)$ in the case of an arbitrary Sylvester nondegenerate cubic surface.

The group in Theorem 4.4 also arose in [MO15] as the group of automorphisms of an Enriques surface whose K3-cover is a quartic surface given by the equation $s_{2}^{2}-t s_{4}=$ $0, t \neq 0,4,36$, where $s_{i}$ denote elementary symmetric functions in variables $t_{0}, t_{1}, t_{2}, t_{3}$. These surfaces belong to a larger family given by the equation $t_{1} s_{2}^{2}+t_{2} s_{4}+t_{3} s_{1} s_{3}=0$, that includes our 1-parameter family when $t_{1}=0$. It was stated in [Dol18] that the proof from [MO15] applies in our case, but its authors have informed us that it does not. The analog of the polytope $P$ in their case is defined by the Coxeter diagram equal to the dual graph of (-2)-curves on Kondo's surface of type $\mathrm{V}$, which also has automorphism group $\mathfrak{S}_{5}$. The surface is the limit (in the appropriate sense) in the family when $t_{2} \rightarrow \infty$ (see [MO15, Remark 2.3]). 
Remark 4.6. - We owe this remark to Matthias Schütt. Consider the family of quartic surfaces in $\mathbb{P}^{3}$ given by the equation

$$
H_{t}:-t s_{4}(x, y, z, w)+s_{1}(x, y, z, w) s_{3}(x, y, z, w)=0,
$$

where $s_{k}(x, y, z, w)$ are elementary symmetric polynomials of degree $k$ in $x, y, z, w$. If the characteristic $p$ of $\mathbb{k}$ is not equal to 2 or 3 , the surface $H_{t}$ is isomorphic to the Hessian surface of a cubic surface with Sylvester parameters $(1,1,1,1, t)$ considered in Theorem 4.2. When $p=2,3$ it is not the Hessian of a cubic surface, but it still contains 10 lines $L_{a b}$ and ten nodes $P_{a b}$ forming the Desargues configuration $\left(10_{3}\right)$. The standard cubic Cremona involution $\tau:(x: y: z: w) \mapsto(1 / x: 1 / y: 1 / z: 1 / w)$ leaves invariant each surface in the family. If $p=2$, the surfaces $S_{t}$ with $t \neq 0, \infty$ are nonsingular, the involution acts freely and the quotient $S_{t}=H_{t} /(\tau)$ is an Enriques surface. There are no Coble surfaces in the family. If $p=3$, the only singular surface in the family is the surface $S_{1}$. It has one singular point $(1: 1: 1: 1)$ and leads to a Coble surface with finite automorphism group isomorphic to $\mathfrak{S}_{5}$. The proofs of Theorems 4.2, 4.3 and 4.4 extend to these families of surfaces. Reinterpreted in terms of the surfaces $H_{t}$, these results therefore apply in all characteristics.

\section{A model of $\operatorname{Aut}(S)$ as a lattice in $\mathrm{PGL}_{2}\left(\mathbb{Q}_{3}\right)$}

This section studies $\operatorname{Aut}(S)$, where $S$ is the Coble surface arising from parameters $\left(\lambda_{0}, \ldots, \lambda_{4}\right)=\left(1,1,1,1, \frac{1}{16}\right)$, under the additional hypothesis that char $\mathbb{k}=0$. In this case there is one new node, so the unique bi-anticanonical divisor is a smooth rational curve $C$, yielding a homomorphism $\operatorname{Aut}(S) \rightarrow \operatorname{Aut}(C) \cong \mathrm{PGL}_{2}(\mathbb{k})$. We completely describe this homomorphism and its image. The main point is that this map is faithful. A side benefit is that this special case is enough to identify the automorphism group of the surface, even when the parameters and ground field characteristic are relaxed to $(1,1,1,1, t \neq 1)$ and char $\mathbb{k} \neq 2,3$. (See the proof of Theorem 4.4.)

Recall from Theorem 4.2 that $\operatorname{Aut}(S)$ is generated by ten involutions $g_{a b}$, where the subscripts vary over the 2 -element subsets of $\{0, \ldots, 4\}$. All we will need to know about them is the following:

(1) they are involutions;

(2) they act nontrivially on $C$;

(3) two commute if their corresponding pairs are disjoint;

(4) if $a, b \neq 4$ then conjugation by $g_{a b}$ permutes the ten involutions by acting on subscripts by the transposition $(a b)$;

(5) these six $g_{a b}$ generate a copy of $\mathfrak{S}_{4}$.

Everything but (2) was established in Section 2. For (2), choose a plane $\Pi$ in $\mathbb{P}^{3}$ containing $P_{a b}$ and the new node $x$, such that the tangent cone of $\Pi \cap H$ consists of two lines. By its definition, $\widetilde{g}_{a b}$ exchanges them. So it acts by a nonscalar on the tangent cone to $H$ at $x$, hence nontrivially on its projectivization. This projectivization is the exceptional divisor of $X$ above $x$, and $C$ is its isomorphic image in $S$. So $g_{a b}$ acts nontrivially on $C$. 
We write $\bar{g}_{a b}$ for the image of $g_{a b}$ in Aut $C$, and $\bar{G}$ for the subgroup of Aut $C$ generated by these ten involutions. Our first description of $\bar{G}$ is as a subgroup of Aut $\mathbb{H}$, where $\mathbb{H}$ is Hamilton's quaternion algebra over $\mathbb{Q}$. By "norm" we mean the reduced norm in the sense of division algebras: $1, i, j$ and $k$ are orthogonal unit vectors. Because $\mathbb{k}$ is algebraically closed, $\mathbb{k} \otimes \mathbb{H}$ is isomorphic to the $2 \times 2$ matrix algebra $M_{2}(\mathbb{k})$. Therefore, any subgroup of Aut $\mathbb{H}$ may be regarded as a subgroup of Aut $M_{2}(\mathbb{k}) \cong \mathrm{PGL}_{2}(\mathbb{k})$. We begin with a complete description of the homomorphism Aut $S \rightarrow$ Aut $C$, in terms of an integral form of $\mathbb{H}$ called the Hurwitz integers and written $\mathcal{H}$. It is defined as the $\mathbb{Z}$-span of the 24 unit norm quaternions $\pm 1, \pm i, \pm j$, $\pm k$ and $\frac{1}{2}( \pm 1 \pm i \pm j \pm k)$.

Theorem 5.1. - There is an isomorphism $C \cong \mathbb{P}^{1}$ under which the four $\bar{g}_{a 4}$ correspond to the images in $\mathrm{PGL}_{2}(\mathbb{k})$ of $\pm i \pm j \pm k$ and the six remaining $\bar{g}_{a b}$ correspond to the images in $\mathrm{PGL}_{2}(\mathbb{k})$ of $\pm i \pm j, \pm j \pm k$ and $\pm k \pm i$.

Proof. - Consider the convex hull in $\operatorname{Im}(\mathbb{H} \otimes \mathbb{R})=\mathbb{R}^{3}$ of $\pm i \pm j \pm k$. It is a cube centered at the origin. The twelve Hurwitz integers $\pm i \pm j, \pm j \pm k$ and $\pm k \pm i$ are the midpoints of its edges. The conjugation action on $\operatorname{Im} \mathbb{H}$ of any one of them is the order 2 rotation that fixes that midpoint. This proves that the subgroup of Aut $\mathbb{H}$ generated by their conjugation actions is the rotation group of the cube. In particular, it is isomorphic to $\mathfrak{S}_{4}$.

Now consider the subgroup $\mathfrak{S}_{4} \subset \operatorname{Aut}(S)$ generated by the $g_{a b}$ with $a, b \neq 4$. It is easy to check that it acts faithfully on the tangent space to $\mathbb{P}^{3}$ at the node $[1: 1: 1: 1:-4]$ of the Hessian surface $H$. It follows that the nontrivial (hence noncentral) elements of $\mathfrak{S}_{4}$ act on this tangent space by non-scalars, which implies that $\mathfrak{S}_{4}$ acts faithfully on the projectivized tangent cone to $H$ at that node. That is, $\mathfrak{S}_{4}$ acts faithfully on $C$.

$\mathrm{PGL}_{2}(\mathbb{k})$ contains a unique conjugacy class of subgroups isomorphic to $\mathfrak{S}_{4}$. So $C$ may be identified with $\mathbb{P}^{1}$ in such a way that these two groups $\mathfrak{S}_{4}$ are identified. Under this identification, the six involutions outside $\mathfrak{A}_{4}$, namely the $\bar{g}_{a b}$ with $a, b \neq 4$, correspond to the six rotations of the cube considered above. That is, these $\bar{g}_{a b}$ are identified with the images in $\mathrm{PGL}_{2}(\mathbb{k})$ of $\pm i \pm j, \pm j \pm k$ and $\pm k \pm i$.

By the hypothesis on how $\mathfrak{S}_{4} \subset \operatorname{Aut}(S)$ permutes $\bar{g}_{04}, \ldots, \bar{g}_{34}$, we know that some $\bar{g}_{a 4}$ centralizes each order 3 subgroup of the rotation group of the cube. For example, $\bar{g}_{04}$ centralizes the subgroup generated by $\bar{g}_{12} \circ \bar{g}_{23}$. The order 3 subgroups are generated by the order 3 rotations around the body-diagonals of the cube. Only one order 2 element of $\mathrm{PGL}_{2}(\mathbb{k})$ centralizes any given order 3 element of $\mathrm{PGL}_{2}(\mathbb{k})$. In our case it is easy to exhibit: the order 2 rotation around that same body-diagonal. That is, by the conjugacy action of one of $\pm i \pm j \pm k$. Therefore the $\bar{g}_{04}, \ldots, \bar{g}_{34}$ act as stated.

Corollary 5.2. - The image $\bar{G}$ of the restriction homomorphism $\operatorname{Aut}(S) \rightarrow$ Aut $(C)$ is conjugate to the subgroup of $\mathrm{SO}(3)$ generated by the order 2 rotations $r_{a b}, 0 \leqslant a, b \leqslant 3$ around the midpoints of the edges of a cube, and the order 2 rotations $r_{a 4}$ around the four body diagonals. 
Remark 5.3. - One can write down explicit matrices for the $\bar{g}_{a b}$ by choosing an isomorphism $\mathcal{H} \otimes \mathbb{k} \cong M_{2}(\mathbb{k})$. For example, if $\mathbb{k}=\mathbb{C}$ then one standard isomorphism is

$$
i \leftrightarrow\left(\begin{array}{cc}
0 & -\mathbf{i} \\
-\mathbf{i} & 0
\end{array}\right) \quad j \leftrightarrow\left(\begin{array}{cc}
0 & -1 \\
1 & 0
\end{array}\right) \quad k \leftrightarrow\left(\begin{array}{cc}
-\mathbf{i} & 0 \\
0 & \mathbf{i}
\end{array}\right)
$$

where $\mathbf{i}=\sqrt{-1} \in \mathbb{C}$. So the matrices for the $\bar{g}_{a b}$ with $a, b \neq 4$ are the signed sums of pairs of these matrices, and the matrices for the $\bar{g}_{a 4}$ are the signed sums of all three. (This gives 20 matrices, but only 10 up to sign.) By direct computation of the restriction homomorphism for the Coble surface, the second author was able, using MAPLE, to find 10 matrices corresponding to $\bar{g}_{a b}$ that generate the same group as do the isometries of a regular tetrahedron and the reflections across its facets. The present proof is less computational, but the original calculation was essential; see the Remark at the end of the paper.

Although pretty, Corollary 5.2's description of $\bar{G}$ is difficult to use because $\bar{G}$ is not discrete in $\mathrm{SO}(3)$. Our next result realizes $\bar{G}$ as a discrete group in $\mathrm{PGL}_{2}\left(\mathbb{Q}_{3}\right)$ rather than $\mathrm{SO}(3)$. Here $\mathbb{Q}_{3}$ is the field of 3-adic rational numbers. The embedding $\bar{G} \rightarrow \mathrm{PGL}_{2}\left(\mathbb{Q}_{3}\right)$ arises as follows.

We write $\mathbf{F}$ for the algebraic group scheme over $\mathbb{Z}$, which to each commutative ring $R$ assigns the group $\operatorname{Aut}(\mathcal{H} \otimes R)$. This is a $\mathbb{Z}$-form of $\mathrm{PGL}_{2}$, in the sense that the functor becomes equal to $\mathrm{PGL}_{2}$ after base changing to any field over which the division algebra $\mathbb{H}$ splits. We claim that $\bar{G} \subset \mathbf{F}\left(\mathbb{Z}\left[\frac{1}{3}\right]\right)$. To see this, note that $\pm i \pm j \pm k$ have norm 3 and so their inverses lie in $\mathcal{H} \otimes \mathbb{Z}\left[\frac{1}{3}\right]$. So their conjugation maps lie in $\mathbf{F}\left(\mathbb{Z}\left[\frac{1}{3}\right]\right)$. The inverses of $\pm i \pm j, \pm j \pm k$ and $\pm k \pm i$ do not lie in $\mathcal{H} \otimes \mathbb{Z}\left[\frac{1}{3}\right]$. But the conjugation maps of these Hurwitz integers do preserve $\mathcal{H}$, hence lie in $\mathbf{F}(\mathbb{Z})$. So $\bar{G} \subset \mathbf{F}\left(\mathbb{Z}\left[\frac{1}{3}\right]\right)$. The embedding $\mathbb{Z}\left[\frac{1}{3}\right] \rightarrow \mathbb{Q}_{3}$ induces an inclusion $\mathbf{F}\left(\mathbb{Z}\left[\frac{1}{3}\right]\right) \rightarrow \mathbf{F}\left(\mathbb{Q}_{3}\right)$. Since $\mathbb{H}$ splits over $\mathbb{Q}_{3}$, we have $\mathbf{F}\left(\mathbb{Q}_{3}\right) \cong \operatorname{PGL}_{2}\left(\mathbb{Q}_{3}\right)$. Putting all of this together yields an embedding $\bar{G} \rightarrow \mathrm{PGL}_{2}\left(\mathbb{Q}_{3}\right)$.

Lemma 5.4. - The groups $\mathbf{F}\left(\mathbb{Z}\left[\frac{1}{3}\right]\right)$ and $\bar{G}$ are discrete in $\mathbf{F}\left(\mathbb{Q}_{3}\right) \cong \mathrm{PGL}_{2}\left(\mathbb{Q}_{3}\right)$.

Proof. - The discreteness of $\mathbb{Z}\left[\frac{1}{3}\right]$ in $\mathbb{R} \times \mathbb{Q}_{3}$ shows that $\mathbf{F}\left(\mathbb{Z}\left[\frac{1}{3}\right]\right)$ is discrete in $\mathbf{F}(\mathbb{R}) \times \mathbf{F}\left(\mathbb{Q}_{3}\right)$. Projecting onto the second factor preserves discreteness because $\mathbf{F}(\mathbb{R}) \cong \mathrm{SO}(3)$ is compact. As a subgroup of $\mathbf{F}\left(\mathbb{Z}\left[\frac{1}{3}\right]\right), \bar{G}$ is also discrete.

Over any $p$-adic field, $\mathrm{PGL}_{2}$ acts properly on a certain tree, which is a standard tool for working with discrete subgroups. We recall the construction over $\mathbb{Q}_{3}$. See $[$ Ser03, Section II.1.1] for background, including the fact that the tree $\mathcal{T}$ we now define is in fact a tree. It has vertex set equal to the set of homothety classes of lattices (rank two $\mathbb{Z}_{3}$-submodules) in $\mathbb{Q}_{3}^{2}$. Note that while PGL $\mathbb{Q}_{2}\left(\mathbb{Q}_{3}\right)$ does not act on $\mathbb{Q}_{3}^{2}$, it does act on the set of homothety classes of lattices. Two vertices are adjacent just if there are lattices representing them, such that one contains the other of index 3. In particular, if a lattice $L$ represents a vertex $v$ of $\mathcal{T}$, then its neighbors in $\mathcal{T}$ correspond to the index 3 sublattices of $L$, or equivalently the 1-dimensional subspaces of $L / 3 L \cong \mathbb{F}_{3}^{2}$. Under $\mathrm{SL}_{2}\left(\mathbb{Q}_{3}\right)$, the vertices fall into two orbits, which are exchanged by any element of $\mathrm{GL}_{2}\left(\mathbb{Q}_{3}\right)$ of determinant 3 .

Lemma 5.5. - Let $A$ be the binary tetrahedral group consisting of the 24 units of $\mathcal{H}$. Its image $\bar{A}$ in $\mathbf{F}(\mathbb{Z})$ lies in $\bar{G}$. 
Proof. - Observe that $(j-k)(i-j)^{-1}=(-1+i+j+k) / 2$. This quaternion is an order 3 element of $A$, and its conjugation map lies in $\bar{G}$ by Theorem 5.1. Conjugating by $\pm i \pm j, \pm j \pm k$ and $\pm k \pm i$ shows that $\bar{G}$ contains the conjugation maps of all eight order 3 elements of $A$, namely $(-1 \pm i \pm j \pm k) / 2$. These generate $A$.

Lemma 5.6. - The binary tetrahedral group $A$ fixes a unique vertex $v$ of $\mathcal{T}$. Each order 3 element of $A$ fixes $v$, exactly one neighbor of $v$, and no other vertices of $\mathcal{T}$.

Proof. - Because $A$ is a finite subgroup of $\mathrm{SL}_{2}\left(\mathbb{Q}_{3}\right)$, it preserves some lattice $L$, for example the $\mathbb{Z}_{3}$-span of the $A$-images of your favorite nonzero vector. We write $v$ for the corresponding vertex of $\mathcal{T}$. By identifying $L$ with $\mathbb{Z}_{3}^{2}$, we identify the $\mathrm{SL}_{2}\left(\mathbb{Q}_{3}\right)$-stabilizer of $L$ with $\mathrm{SL}_{2}\left(\mathbb{Z}_{3}\right)$. Because the kernel of $\mathrm{SL}_{2}\left(\mathbb{Z}_{3}\right) \rightarrow \mathrm{SL}_{2}\left(\mathbb{F}_{3}\right)$ is a pro-3 group, the normal subgroup $Q_{8}$ of $A$ maps faithfully to $\mathrm{SL}_{2}\left(\mathbb{F}_{3}\right)$. Since every $\mathbb{Z} / 3$ subgroup of $A$ acts nontrivially on $Q_{8}$, it also maps faithfully. Therefore the composition $A \rightarrow \mathrm{SL}_{2}\left(\mathbb{Z}_{3}\right) \rightarrow \mathrm{SL}_{2}\left(\mathbb{F}_{3}\right)$ is injective. It is even an isomorphism, because $\left|\mathrm{SL}_{2}\left(\mathbb{F}_{3}\right)\right|=24$.

We have shown that $A$ acts on $L / 3 L \cong \mathbb{F}_{3}^{2}$ as $\mathrm{SL}_{2}\left(\mathbb{F}_{3}\right)$. In particular, it permutes the four 1-dimensional $\mathbb{F}_{3}$-subspaces as the alternating group of degree 4 . It follows that $A$ fixes no neighbor of $v$ (hence no point of $\mathcal{T}$ other than $v$ ), and that each order 3 element of $A$ fixes exactly one neighbor of $v$.

It remains to show that no vertex at distance 2 from $v$ is fixed by any order 3 element of $A$. Each such vertex is represented by a lattice $M$ having index 9 in $L$. Furthermore, $L / M$ cannot be isomorphic to $(\mathbb{Z} / 3)^{2}$, because that would force $M=3 L$, which corresponds to the vertex $v$ rather than to a vertex at distance 2 . Therefore $L / M \cong \mathbb{Z} / 9$. It follows that the vertices at distance 2 correspond to the 12 subgroups $\mathbb{Z} / 9$ of $L / 9 L \cong(\mathbb{Z} / 9)^{2}$. It suffices to show that no order 3 element of $A$ preserves any one of them. This follows from the claim: every order 3 element of $\mathrm{SL}_{2}(\mathbb{Z} / 9)$, that preserves some $\mathbb{Z} / 9 \subset(\mathbb{Z} / 9)^{2}$, lies in the kernel of $\mathrm{SL}_{2}(\mathbb{Z} / 9) \rightarrow$ $\mathrm{SL}_{2}\left(\mathbb{F}_{3}\right)$.

To prove the claim, we use the fact that all the $\mathbb{Z} / 9$ 's are $\mathrm{SL}_{2}(\mathbb{Z} / 9)$-equivalent, so it is enough to examine the order 3 elements in the stabilizer of the $\mathbb{Z} / 9$ generated by $\left(\begin{array}{l}1 \\ 0\end{array}\right)$. This stabilizer is the semidirect product $\langle\tau\rangle \rtimes\langle\sigma\rangle$, where $\tau=\left(\begin{array}{ll}1 & 1 \\ 0 & 1\end{array}\right)$ has order 9 and $\sigma=\left(\begin{array}{cc}2 & 0 \\ 0 & 1 / 2\end{array}\right)$ has order 6 . One can check that $\sigma \tau \sigma^{-1}=\tau^{4}$. We must show that every order 3 element $x \in\langle\tau\rangle \rtimes\langle\sigma\rangle$ has trivial image in $\mathrm{SL}_{2}\left(\mathbb{F}_{3}\right)$. It is clear that every order 3 element lies in $\langle\tau\rangle \rtimes\left\langle\sigma^{2}\right\rangle$. If $x=\tau^{i}$ then the relation $x^{3}=1$ forces $3 \mid i$. If $x=\tau^{i} \sigma^{ \pm 2}$ then the relation $x^{3}=1$ boils down to $\tau^{273 i}=1$, which again forces $3 \mid i$. We have proven $x \in\left\langle\tau^{3}\right\rangle \rtimes\left\langle\sigma^{2}\right\rangle$. This implies our claim, because $\tau^{3}$ and $\sigma^{2}$ map to the identity of $\mathrm{SL}_{2}\left(\mathbb{F}_{3}\right)$.

THEOREM 5.7. -

(1) The subgroup of $\operatorname{Aut}(C) \cong \mathrm{PGL}_{2}(\mathbb{k})$ generated by $\bar{g}_{04}, \ldots, \bar{g}_{34}$ is the free product $\left\langle\bar{g}_{04}\right\rangle * \cdots *\left\langle\bar{g}_{34}\right\rangle$ of four copies of $\mathbb{Z} / 2$. It acts simply transitively on the vertices of $\mathcal{T}$.

(2) The image $\bar{G}$ of $\operatorname{Aut}(S)$ in $\operatorname{Aut}(C) \cong \mathrm{PGL}_{2}(\mathbb{k})$ is the semidirect product of the group from (1) by the symmetric group $\mathfrak{S}_{4}$, permuting the free factors $\mathbb{Z} / 2$ in the obvious way.

In particular, the natural map $\operatorname{Aut}(S) \rightarrow \operatorname{Aut}(C)$ is injective. 


\section{Proof.}

(1). - Even though $\bar{G}$ was defined as a subgroup of $\operatorname{Aut}(C) \cong \mathrm{PGL}_{2}(\mathbb{k})$, we will continue to work with it as a subgroup of $\mathrm{PGL}_{2}\left(\mathbb{Q}_{3}\right)$. We continue to write $v$ for the unique vertex of $\mathcal{T}$ fixed by the binary tetrahedral group $A$. Each of $\pm i \pm j \pm k$ has norm 3 in $\mathcal{H}$, hence determinant 3 when regarded as an element of $\mathrm{GL}_{2}\left(\mathbb{Q}_{3}\right)$. Therefore each $\bar{g}_{a 4}$ exchanges the two $\mathrm{SL}_{2}\left(\mathbb{Q}_{3}\right)$-orbits of vertices of $\mathcal{T}$. In particular, each $\bar{g}_{a 4}$ moves $v$ to some other vertex.

Next, $\bar{g}_{a 4}$ centralizes some order 3 subgroup $\Theta_{a}$ of $\mathfrak{S}_{4} \subset \bar{G}$. The image of $A$ in $\mathrm{PGL}_{2}\left(\mathbb{Q}_{3}\right)$ contains all the order 3 subgroups of $\mathfrak{S}_{4}$. Therefore Lemma 5.6 shows that $\Theta_{a}$ fixes the vertex $v$ and one of its neighbors, but no other vertices of $\mathcal{T}$. Since $\bar{g}_{a 4}$ centralizes $\Theta_{a}$, it preserves this set of two vertices. In the previous paragraph we saw that $\bar{g}_{a 4}$ moves $v$ to some other vertex. Therefore $\bar{g}_{a 4}$ exchanges $v$ with the neighbor fixed by $\Theta_{a}$. The midpoint $m_{a}$ of the edge joining these vertices is the only fixed point of $\bar{g}_{a 4}$ in $\mathcal{T}$. We think of $\bar{g}_{a 4}$ as acting on $\mathcal{T}$ by a sort of reflection, whose mirror consists of the single point $m_{a}$. Each of $\bar{g}_{04}, \ldots, \bar{g}_{34}$ centralizes a different order 3 subgroup of $\mathfrak{S}_{4}$, so $m_{0}, \ldots, m_{3}$ are the midpoints of the four edges emanating from $v$.

This suggests that the union $D$ of the four half-edges from $v$ to $m_{0}, \ldots, m_{3}$ should be a fundamental domain for the action of $\left\langle\bar{g}_{04}, \ldots, \bar{g}_{34}\right\rangle$ on $\mathcal{T}$. This can be verified by using Poincaré's Polyhedron Theorem. Standard references, such as [Mas88, Section IV.H], only develop this theorem for groups acting on manifolds. So we sketch the proof in our situation, which is actually much simpler than the general manifold case.

We set $\bar{H}=\left\langle\bar{g}_{04}, \ldots, \bar{g}_{34}\right\rangle$. (There is no group $H$; the bar merely reminds us that $\bar{H}$ lies in $\bar{G}$.) We also introduce formal symbols $\widetilde{g}_{04}, \ldots, \widetilde{g}_{34}$, each generating a copy of $\mathbb{Z} / 2$, and set

$$
\widetilde{H}=\left\langle\widetilde{g}_{04}\right\rangle * \cdots *\left\langle\widetilde{g}_{34}\right\rangle \cong(\mathbb{Z} / 2) *(\mathbb{Z} / 2) *(\mathbb{Z} / 2) *(\mathbb{Z} / 2) .
$$

We write elements of $\widetilde{H}$ with tildes, and indicate the natural map $\widetilde{H} \rightarrow \bar{H}$ by converting tildes to bars. We think of $\widetilde{H} \times D$ as a disjoint union of copies of $D$ indexed by $\widetilde{h} \in \widetilde{H}$. There is a natural $\widetilde{H}$-action on this union, with $\widetilde{g} \in \widetilde{H}$ sending $(\widetilde{h}, d)$ to $(\widetilde{g} \widetilde{h}, d)$. The map $\widetilde{H} \times D \rightarrow \mathcal{T}$ defined by $(\widetilde{h}, d) \mapsto \bar{h}(d)$ is compatible with the natural map $\widetilde{H} \rightarrow \bar{H}$ and the $\widetilde{H}$ - and $\bar{H}$-actions on $\widetilde{H} \times D$ and $\mathcal{T}$. We glue the copies of $D$ together to form a connected graph $\widetilde{\mathcal{T}}$, by identifying $\left(\widetilde{h}, m_{a}\right)$ with $\left(\widetilde{h} \widetilde{g}_{a 4}, m_{a}\right)$, for every $\widetilde{h} \in \widetilde{H}$ and $a=0, \ldots, 3$. The gluing is compatible with the $\widetilde{H}$-action, so $\widetilde{H}$ acts on $\widetilde{\mathcal{T}}$. The gluing is also compatible with the projection $\widetilde{H} \times D \rightarrow \mathcal{T}$, which therefore descends to a map $\widetilde{\mathcal{T}} \rightarrow \mathcal{T}$. This map is compatible with $\widetilde{H} \rightarrow \bar{H}$ and the $\widetilde{H}$ - and $\bar{H}$-actions. It is easy to check that $\widetilde{\mathcal{T}} \rightarrow \mathcal{T}$ is a covering map, hence a homeomorphism. It follows that $\widetilde{H} \rightarrow \bar{H}$ must be an isomorphism. The simple transitivity of $\widetilde{H}$ on the vertices $(\widetilde{h}, v)$ of $\widetilde{\mathcal{T}}$ is obvious, so $\bar{H}$ acts simply transitively on the vertices of $\mathcal{T}$.

(2). - Having proven (1), we know that $\bar{G}$ is generated by $\mathfrak{S}_{4}$ and the free product of four copies of $(\mathbb{Z} / 2)$, with the first group normalizing the second. To establish the semidirect product decomposition we must show that these groups meet trivially. As a finite subgroup of the free product, the intersection is conjugate into one of 
the free factors by [Ser03, Theorem 8 of Section I.4.3]. Therefore it has order $\leqslant 2$. But the intersection is also normal in $\mathfrak{S}_{4}$, which has no normal subgroups of order 2. Therefore the intersection is trivial.

TheOrem 5.8. - The group $\bar{G}$ coincides with $\mathbf{F}\left(\mathbb{Z}\left[\frac{1}{3}\right]\right)$, which is maximal among discrete subgroups of $\mathrm{PGL}_{2}\left(\mathbb{Q}_{3}\right)$.

Proof. - Because $\mathbf{F}\left(\mathbb{Z}\left[\frac{1}{3}\right]\right)$ is discrete and contains $\bar{G}$, it suffices to show that $\bar{G}$ is maximal among discrete subgroups of $\mathrm{PGL}_{2}\left(\mathbb{Q}_{3}\right)$. So suppose $\Gamma$ is a discrete subgroup that contains it. Because $\bar{G}$ acts transitively on the vertices of $\mathcal{T}, \Gamma$ is generated by $\bar{G}$ and the $\Gamma$-stabilizer of $v$. The latter is finite, by discreteness. It contains the $\bar{G}$-stabilizer $\mathfrak{S}_{4}$ of $v$. Since $\mathfrak{S}_{4}$ is maximal among finite subgroups of $\mathrm{PGL}_{2}$, over any field of characteristic 0 , the $\Gamma$-stabilizer is the same as the $\bar{G}$-stabilizer. So $\Gamma=\bar{G}$.

Theorem 5.7 has an appealing consequence:

TheOREM 5.9. - Let $T$ be a regular tetrahedron in Euclidean 3-space $\mathbb{R}^{3}$. Then the group of isometries generated by the automorphisms of $T$ and the reflections across its facets is

$$
((\mathbb{Z} / 2) *(\mathbb{Z} / 2) *(\mathbb{Z} / 2) *(\mathbb{Z} / 2)) \rtimes \operatorname{Aut}(T) .
$$

Proof. - It suffices to show that the image of this group in $\mathrm{O}(3)$ has this structure. Combining Corollary 5.2 and Theorem 5.7 shows that the group $\bar{G} \subset \mathrm{SO}(3)$ generated by the rotations $r_{a b}(a, b=0, \ldots, 3)$ around the midpoints of the edges of the cube, and the rotations $r_{a 4}$ around the body diagonals of the cube, has this structure. Replacing each $r_{a b}$ by $-r_{a b}$ replaces each $g \in \bar{G}$ by $\pm g$, and therefore does not change the isomorphism type of the subgroup of $\mathrm{O}(3)$ they generate. (This uses $-1 \notin \bar{G}$.)

We identify $T$ with one of the two regular tetrahedra inscribed in the cube. (That is, $T$ is the convex hull of 4 vertices of the cube, no two adjacent.) Then the planes through the origin, perpendicular to the body diagonals, are parallel to the facets of $T$. Therefore the $-r_{a 4}$ are the reflections across them. And the $-r_{a b}$ with $a, b \leqslant 3$ are reflections that generate the isometry group of $T$.

An amusing way to interpret this is that you can reflect $T$ across a facet, and then reflect that image of $T$ across one of its facets, and so on. Imagine doing this and then challenging your friend to return the tetrahedron to its original position by further reflections. The only solution is to retrace your sequence of reflections. This has the same flavor as Rich Schwartz's game "Lucy and Lily" [Sch02], which uses a regular pentagon in the plane in place of our tetrahedron.

In fact, the entire paper grew backwards from Theorem 5.9. The explicit matrix computations referred to in Remark 5.3 identified $\bar{G}$ with the group from this theorem. The problem of identifying the image led to the discrete subgroup of $\operatorname{PGL}_{2}\left(\mathbb{Q}_{3}\right)$. Having identified $\bar{G}$, it was natural to wonder whether it was the full and faithful image of $\operatorname{Aut}(S)$, which led to the computation of the nef cone. 


\section{BIBLIOGRAPHY}

[Bou81] Nicolas Bourbaki, Groupes et algèbres de Lie, Chapitres 4, 5 et 6, Élements de Mathématique, Masson, 1981. $\uparrow 1151$

[CD89] François Cossec and Igor V. Dolgachev, Enriques surfaces. I., Progress in Mathematics, vol. 76, Birkhäuser, 1989. $\uparrow 1135$

[Cob39] Arthur B. Coble, Cremona transformations with an invariant rational sextic, Bull. Am. Math. Soc. 45 (1939), no. 4, 285-288. $\uparrow 1134$

[Cob82] _ _ Algebraic geometry and theta functions, Colloquium Publications, vol. 10, American Mathematical Society, 1982, reprint of the 1929 edition. $\uparrow 1134$

[DO19] Tien-Cuong Dinh and Keiji Oguiso, A surface with discrete and non-finitely generated automorphism group, Duke Math. J. 168 (2019), no. 6, 941-966. ^1134

[Dol08] Igor V. Dolgachev, Reflection groups in algebraic geometry, Bull. Am. Math. Soc. 45 (2008), no. 1, 1-60. $\uparrow 1145,1148$

[Dol12] _ Classical algebraic geometry. A modern view, Cambridge University Press, 2012. $\uparrow 1135,1136,1142$

[Dol16] _ A brief introduction to Enriques surfaces, Development of moduli theory, Kyoto 2013, Advanced Studies in Pure Mathematics, vol. 69, Mathematical Society of Japan, 2016, pp. 1-32. $\uparrow 1145$

[Dol18] _ Salem numbers and Enriques surfaces, Exp. Math. 27 (2018), no. 3, $287-301$. $\uparrow 1134,1136,1143,1151$

[DZ01] Igor V. Dolgachev and De-Qi Zhang, Coble rational surfaces, Am. J. Math. 123 (2001), no. $1,79-114 . \uparrow 1139$

[Kon86] Shigeyuki Kondō, Enriques surfaces with finite automorphism groups, Jap. J. Math. 12 (1986), no. 2, 191-282. $\uparrow 1135,1145,1148$

[Lei18] John Leisieutre, A projective variety with discrete, non-finitely generated automorphism group, Invent. Math. 212 (2018), no. 1, 189-211. $\uparrow 1134$

[Mas88] Bernard Maskit, Kleinian Groups, Grundlehren der Mathematischen Wissenschaften, vol. 287, Springer, 1988. $\uparrow 1156$

[MO15] Shigeru Mukai and Hisanori Ohashi, The automorphism groups of Enriques surfaces covered by symmetric quartic surfaces, Recent advances in algebraic geometry, London Mathematical Society Lecture Note Series, vol. 417, Cambridge University Press, 2015, pp. 307-320. $\uparrow 1151$

[Sch02] Richard Evan Schwartz, Lucy and Lily: A Game of Geometry and Number Theory, Am. Math. Mon. 109 (2002), no. 1, 13-20. $\uparrow 1157$

[Ser03] Jean-Pierre Serre, Trees, Springer Monographs in Mathematics, Springer, 2003. $\uparrow 1154$, 1157

[Shi19] Ichiro Shimada, On an Enriques surface associated with a quartic Hessian surface, Can. J. Math. 71 (2019), no. 1, 213-246. $\uparrow 1135,1145,1151$

[Sil09] Joseph Hillel Silverman, The arithmetic of elliptic curves, second ed., Graduate Texts in Mathematics, vol. 106, Springer, 2009. $\uparrow 1142$

[Vin71] Èrnest Borisovich Vinberg, Discrete linear groups generated by reflections, Math. USSR, Izv. 5 (1971), no. 5, 1083-1119. ^1145, 1151

[Vin72] _ On the groups of unit elements of certain quadratic forms, Math. USSR, Sb. 16 (1972), 17-35. $\uparrow 1148$ 
Manuscript received on 19th October 2018, revised on 24th August 2019, accepted on 23rd October 2019.

Recommended by Editor S. Cantat. Published under license CC BY 4.0.

(c) (i)

This journal is a member of Centre Mersenne.

MENTRE

Daniel ALLCOCK

Department of Mathematics, University of Texas

Austin, (USA)

allcock@math.utexas.edu

Igor DOLGACHEV

Department of Mathematics, University of Michigan

Ann Arbor, (USA)

idolga@umich.edu 